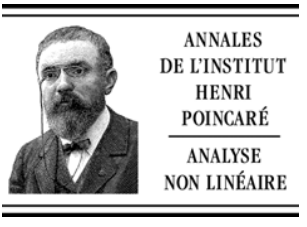

www.elsevier.com/locate/anihpc

\title{
H-surface index formula
}

\author{
Ruben Jakob ${ }^{1}$ \\ Mathematisches Institut der Universität, Bonn, Germany
}

Received 6 June 2004; accepted 6 October 2004

Available online 23 May 2005

\begin{abstract}
We construct an additive index $\mathcal{I}$ on the set of compact "parts" of the set $\mathcal{H}_{H}(\Gamma)$ of "small" H-surfaces $\left(|H|<\frac{1}{2}\right)$ that are spanned into a simple closed polygon $\Gamma \subset \mathbb{R}^{3}$ with $N+3$ vertices $(N \geqslant 1)$ by a combination of Heinz' and Hildebrandt's examinations of H-surfaces and Dold's fixed point theory. We obtain that the index of $\mathcal{H}_{H}(\Gamma)$ is always 1, independent of $H$ and $\Gamma$. Moreover we compute that the Čech cohomology $\check{H}(\mathcal{P})$ of a part $\mathcal{P}$ that minimizes the $\mathrm{H}$-surface functional $\mathcal{E}^{H}$ locally is non-trivial at most in degrees $0, \ldots, N-1$ and there even finitely generated, which implies the finiteness of the number of connected components of $\mathcal{P}$ in particular. Finally the index of such an " $\not \mathcal{E}$-minimizing" part reveals to coincide with its Čech-Euler characteristic, which yields a variant of the mountain-pass-lemma.

(C) 2005 L'Association Publications de l'Institut Henri Poincaré. Published by Elsevier B.V. All rights reserved
\end{abstract}

\section{Introduction and main result}

Let $\Gamma$ be some closed piecewise linear Jordan curve $\Gamma \subset \mathbb{D}^{3}:=\left\{x \in \mathbb{R}^{3}|| x \mid \leqslant 1\right\}$ with $N+3$ vertices $(N \in \mathbb{N})$

$$
\left(P_{0}, A_{1}, \ldots, A_{l} ; P_{1} ; A_{l+1}, \ldots, A_{m} ; P_{2} ; A_{m+1}, \ldots, A_{N}\right),
$$

where the three vertices $P_{0}, P_{1}, P_{2}$ and the indices $0 \leqslant l \leqslant m \leqslant N$ are fixed. We consider the (Plateau-) class $\mathcal{C}_{1}^{*}(\Gamma)$ of surfaces $X \in H^{1,2}\left(B, \mathbb{R}^{3}\right) \cap C^{0}\left(\bar{B}, \mathbb{R}^{3}\right), B:=B_{1}(0) \subset \mathbb{R}^{2}$, that are spanned into $\Gamma$, i.e. whose boundary values $\left.X\right|_{\partial B}: \mathbb{S}^{1} \rightarrow \Gamma$ are weakly monotonic mappings with degree equal to 1 , satisfying a three-point-condition:

$$
\left.X\right|_{\partial B}\left(\mathrm{e}^{\mathrm{i} \psi_{k}}\right)=P_{k}, \quad \psi_{k}:=k \frac{2 \pi}{3}, k=0,1,2,
$$

and that are contained in the closed unit ball $\mathbb{D}^{3}$, i.e. $\|X\|_{C^{0}(\bar{B})} \leqslant 1$. We endow $\mathcal{C}_{1}^{*}(\Gamma)$ with the norm $\|\cdot\|_{H^{1,2} \cap C^{0}}:=$ $\|\cdot\|_{C^{0}(\bar{B})}+\|\cdot\|_{H^{1,2}(B)}$. Moreover we consider the subspace $\mathcal{H}_{H}(\Gamma) \subset\left(\mathcal{C}_{1}^{*}(\Gamma),\|\cdot\|_{H^{1,2} \cap C^{0}}\right)$ of all "small" H-surfaces, i.e. classical solutions $X \in \mathcal{C}_{1}^{*}(\Gamma) \cap C^{2}\left(B, \mathbb{R}^{3}\right)$ of the differential equations

E-mail address: ruben@math.uni-bonn.de (R. Jakob).

1 It is a pleasure for the author to thank Prof. Dr. S. Hildebrandt for his support.

0294-1449/\$ - see front matter C 2005 L'Association Publications de l'Institut Henri Poincaré. Published by Elsevier B.V. All rights reserved doi:10.1016/j.anihpc.2004.10.008 


$$
\begin{aligned}
& \triangle X=2 H\left(X_{u} \wedge X_{v}\right) \quad \text { on } B, \\
& \left|X_{u}\right|^{2}=\left|X_{v}\right|^{2}, \quad\left\langle X_{u}, X_{v}\right\rangle=0 \quad \text { on } B,
\end{aligned}
$$

for some arbitrarily fixed $H \in\left(-\frac{1}{2}, \frac{1}{2}\right)$. We note that Eqs. (3) are just the Euler-Lagrange equations of the "H-surface functional" $\mathcal{E}^{H}: H^{1,2}\left(B, \mathbb{R}^{3}\right) \cap L^{\infty}\left(B, \mathbb{R}^{3}\right) \rightarrow \mathbb{R}$, given by

$$
\mathcal{E}^{H}(X):=\int_{B}|\nabla X|^{2}+\frac{4 H}{3}\left\langle X, X_{u} \wedge X_{v}\right\rangle \mathrm{d} u \mathrm{~d} v .
$$

For some fixed polygon $\Gamma$ and $H \in\left(-\frac{1}{2}, \frac{1}{2}\right)$ Heinz [7] constructed a map

$$
\psi: T \rightarrow\left(\mathcal{C}_{1}^{*}(\Gamma),\|\cdot\|_{H^{1,2} \cap C^{0}}\right)
$$

on a convex, open, bounded subset $T \subset \mathbb{R}^{N}$ (where $N+3$ was the number of vertices of $\Gamma$ ), whose following crucial properties shall be proved in this paper using Heinz' isoperimetric inequality in [8], his boundary regularity theorem in [10] combined with an idea of Hildebrandt [12] and the author's generalizations ([15] resp. [16]) of Courant's fundamental ideas in $[2,3]$ :

\section{Reduction theorem.}

(i) $\psi$ and $f:=\mathcal{E}^{H} \circ \psi$ are continuous on $T$.

(ii) We have even $f=\mathcal{E}^{H} \circ \psi \in C^{1}(T, \mathbb{R})$.

(iii) For every sequence $\left\{\tau^{n}\right\}_{n \in \mathbb{N}}$ with $\operatorname{dist}\left(\tau^{n}, \partial T\right) \rightarrow 0$ there holds

$$
f\left(\tau^{n}\right) \rightarrow \infty \text { for } n \rightarrow \infty .
$$

(iv) The restriction

$$
\left.\psi\right|_{K(f)}: K(f) \stackrel{\cong}{\rightrightarrows} \mathcal{H}_{H}(\Gamma)
$$

yields a homeomorphism between the compact set $K(f)$ of critical points of $f$ and $\left(\mathcal{H}_{H}(\Gamma),\|\cdot\|_{H^{1,2} \cap C^{0}}\right)$.

We furthermore define the two following notions in

Definition 1.1. (i) A compact subset $\mathcal{P} \subseteq \mathcal{H}_{H}(\Gamma)$ is termed a part (of $\mathcal{H}_{H}(\Gamma)$ ) if $\mathcal{P}$ has an open neighborhood $\mathcal{U}$ in $\left(\mathcal{C}_{1}^{*}(\Gamma),\|\cdot\|_{H^{1,2} \cap C^{0}}\right)$ which satisfies $\mathcal{P}=\mathcal{U} \cap \mathcal{H}_{H}(\Gamma)$, i.e. which separates $\mathcal{P}$ from the complement $\mathcal{H}_{H}(\Gamma) \backslash \mathcal{P}$, and we set

$$
\mathbf{K}_{H}(\Gamma):=\left\{\mathcal{P} \subseteq \mathcal{H}_{H}(\Gamma) \mid \mathcal{P} \text { is a part }\right\}
$$

(ii) An $\mathcal{E}^{H}$-minimizing part $\mathcal{P}(\neq \emptyset)$ of $\mathcal{H}_{H}(\Gamma)$ is characterized by the two following additional properties:

(1) $\mathcal{E}^{H}(X) \equiv$ const. $(\mathcal{P}) \quad \forall X \in \mathcal{P}$,

(2) there exists an open neighborhood $\mathcal{U}$ of $\mathcal{P}$ in $\left(\mathcal{C}_{1}^{*}(\Gamma),\|\cdot\|_{H^{1,2} \cap C^{0}}\right)$ such that

$$
\mathcal{E}^{H}(X) \geqslant \text { const. }(\mathcal{P}) \quad \forall X \in \mathcal{U} \text {. }
$$

Now we can state the

Main theorem. To any closed polygon $\Gamma \subset \mathbb{D}^{3}$ and $H \in\left(-\frac{1}{2}, \frac{1}{2}\right)$ one can assign an (H-surface-) index

$$
\mathcal{I}: \mathbf{K}_{H}(\Gamma) \rightarrow \mathbb{Z}
$$

with the following properties: 
(i) $\mathcal{I}\left(\mathcal{H}_{H}(\Gamma)\right)=1$, independent of $H$ and $\Gamma$.

(ii) $\mathcal{I}$ is additive on $\mathbf{K}_{H}(\Gamma)$, i.e. any decomposition $\mathcal{P}=\bigsqcup_{j=1}^{m} \mathcal{P}_{j} \in \mathbf{K}_{H}(\Gamma)(m \geqslant 1)$ yields

$$
\mathcal{I}(\mathcal{P})=\sum_{j=1}^{m} \mathcal{I}\left(\mathcal{P}_{j}\right)
$$

(iii) Moreover the Čech cohomology $\check{H}(\mathcal{P})$ of an $\mathcal{E}^{H}$-minimizing part $\mathcal{P} \subseteq \mathcal{H}_{H}(\Gamma)$ is non-trivial at most in degrees $0, \ldots, N-1$ and there even finitely generated, $\mathcal{P}$ consists of only finitely many connected components and its H-surface-index coincides with its Čech-Euler-characteristic:

$$
\mathcal{I}(\mathcal{P})=\sum_{i=0}^{N-1}(-1)^{i} \operatorname{dim}_{\mathbb{Q}} \check{H}^{i}(\mathcal{P}, \mathbb{Q})=: \check{\chi}(\mathcal{P}, \mathbb{Q}) .
$$

This theorem immediately implies the following

\section{Corollaries.}

(i) $\mathcal{H}_{H}(\Gamma) \neq \varnothing$.

(ii) A variant of the mountain-pass-lemma: If there exist $m \geqslant 2$ different $\mathcal{E}^{H}$-minimizing parts $\mathcal{P}_{j}$ in $\mathcal{H}_{H}(\Gamma)$ with $\sum_{j=1}^{m} \check{\chi}\left(\mathcal{P}_{j}, \mathbb{Q}\right) \neq 1$, then the complement $\mathcal{H}_{H}(\Gamma) \backslash \bigcup_{j=1}^{m} \mathcal{P}_{j}$ is not empty. This situation is encountered especially if there exist $m \geqslant 2$ homotopy equivalent $\mathcal{E}^{H}$-minimizing parts $\mathcal{P}_{j}$ in $\mathcal{H}_{H}(\Gamma)$. If in particular these parts $\mathcal{P}_{j}$ are points, i.e. isolated $H$-surfaces $X_{1}, \ldots, X_{m}$ which are local minimizers of $\mathcal{E}^{H}$ in $\left(\mathcal{C}_{1}^{*}(\Gamma),\|\cdot\|_{H^{1,2} \cap C^{0}}\right)$, then the $H$-surface-index of the complementary part $\mathcal{P}:=\mathcal{H}_{H}(\Gamma) \backslash\left\{X_{1}, \ldots, X_{m}\right\}$ amounts to

$$
\mathcal{I}(\mathcal{P})=1-m<0 .
$$

The author would like to point out the similarity of statement (i) of the main theorem to the result of Tromba's papers [21] resp. [22], where Tromba constructs a "minimal surface index" which can be assigned to isolated minimal surfaces spanning a wire $\Gamma \in H^{r, 2}\left(\partial B, \mathbb{R}^{n}\right)(r>18, n \geqslant 3)$ that are non-degenerate in two different senses depending on the two cases $n>3$ resp. $n=3$. The fundamental tool for his "Index formula" of [21,22] yields the deep Index Theorem of Böhme and himself [1] which guarantees that at least for an open, dense subset of boundary curves $\Gamma$ in $H^{r, 2}\left(\partial B, \mathbb{R}^{n}\right)$ (so-called generic curves) the set $\mathcal{M}(\Gamma)$ of all minimal surfaces spanning $\Gamma$ indeed consists of isolated and non-degenerate points in the two cases $n>3$ resp. $n=3$. One should also compare our main theorem to Struwe's achievements in [19], where he develops a complete Morse theory for the description of $\mathcal{M}(\Gamma)$, provided $\Gamma \subset \mathbb{R}^{n}(n \geqslant 2)$ is a $C^{5}$-regular boundary curve that spans only minimal surfaces which are non-degenerate critical points of the Dirichlet integral, which is guaranteed at least for generic wires $\Gamma \subset \mathbb{R}^{n}$ for $n \geqslant 4$ by the Index Theorem [1] of Böhme and Tromba. Finally one should notice the similarity of Corollary (ii) to Struwe's resp. Imbusch's mountain-pass-lemma in [20], p. 51, resp. [14], p. 17, which also does not require the existence of different strict local minimizers of the considered functional (there it is the Dirichlet integral) on the class of admitted surfaces spanned into the boundary curve.

\section{Fundamental properties of $\mathcal{E}^{H}$ and H-surfaces}

For $X \in H^{1,2}\left(B, \mathbb{R}^{3}\right) \cap L^{\infty}\left(B, \mathbb{R}^{3}\right)$ and any $\mathcal{L}^{2}$-measurable subset $B^{\prime} \subseteq B$ we set

$$
\mathcal{D}_{B^{\prime}}(X):=\frac{1}{2} \int_{B^{\prime}}|\nabla X|^{2} \mathrm{~d} u \mathrm{~d} v, \quad \mathcal{F}_{B^{\prime}}(X):=\int_{B^{\prime}}\left\langle X, X_{u} \wedge X_{v}\right\rangle \mathrm{d} u \mathrm{~d} v \quad \text { and }
$$




$$
\mathcal{E}_{B^{\prime}}^{H}(X):=2 \mathcal{D}_{B^{\prime}}(X)+\frac{4 H}{3} \mathcal{F}_{B^{\prime}}(X) \text { for }|H|<\frac{1}{2},
$$

and we shall use the abbreviations $\mathcal{D}(X):=\mathcal{D}_{B}(X), \mathcal{F}(X):=\mathcal{F}_{B}(X)$ and $\mathcal{E}^{H}(X):=\mathcal{E}_{B}^{H}(X)$. At first we have for $X \in H^{1,2}\left(B, \mathbb{R}^{3}\right) \cap L^{\infty}\left(B, \mathbb{R}^{3}\right)$ with $\|X\|_{L^{\infty}(B)} \leqslant 1$ due to $\left|X_{u} \wedge X_{v}\right| \leqslant \frac{1}{2}|\nabla X|^{2}$ the estimates

$$
\left(1-\frac{2}{3}|H|\right) 2 \mathcal{D}_{B^{\prime}}(X) \leqslant \mathcal{E}_{B^{\prime}}^{H}(X) \leqslant\left(1+\frac{2}{3}|H|\right) 2 \mathcal{D}_{B^{\prime}}(X)
$$

on any $\mathcal{L}^{2}$-measurable subset $B^{\prime} \subseteq B$. By these inequalities one obtains as in Lemma 3.4 in [11] the lower semicontinuity of $\mathcal{E}^{H}$ with respect to weak convergence in $H^{1,2}\left(B, \mathbb{R}^{3}\right)$ :

Lemma 2.1. For a sequence $\left\{X_{j}\right\} \subset H^{1,2}\left(B, \mathbb{R}^{3}\right) \cap L^{\infty}\left(B, \mathbb{R}^{3}\right)$ with $X_{j} \rightarrow X$ in $H^{1,2}\left(B, \mathbb{R}^{3}\right)$ and $\left\|X_{j}\right\|_{L^{\infty}(B)} \leqslant 1$ we have

$$
\mathcal{E}^{H}(X) \leqslant \liminf _{j \rightarrow \infty} \mathcal{E}^{H}\left(X_{j}\right)
$$

Analogously one can prove the same statement for the functional $\mathcal{G}^{H}:=\mathcal{D}+\frac{4 H}{3} \mathcal{F}$ (for $|H|<1 / 2$ ). By these tools one easily infers (see [17], Lemma 2.2)

Lemma 2.2. Let $\left\{X_{j}\right\}$ be a sequence in $H^{1,2}\left(B, \mathbb{R}^{3}\right) \cap L^{\infty}\left(B, \mathbb{R}^{3}\right)$ with $\left\|X_{j}\right\|_{L^{\infty}(B)} \leqslant 1 \forall j \in \mathbf{N}$,

$$
\begin{aligned}
& \mathcal{E}^{H}\left(X_{j}\right) \rightarrow \mathcal{E}^{H}(X) \text { and } \\
& X_{j} \rightarrow X \text { in } H^{1,2}\left(B, \mathbb{R}^{3}\right) \text { for } j \rightarrow \infty,
\end{aligned}
$$

for some surface $X \in H^{1,2}\left(B, \mathbb{R}^{3}\right)$, then there holds:

$$
\mathcal{D}\left(X_{j}\right) \rightarrow \mathcal{D}(X) \quad \text { for } j \rightarrow \infty .
$$

Now we consider the Dirichlet problem for $\mathcal{E}^{H}$ and prescribed boundary values $r:=\left.X\right|_{\partial B}$ of a surface $X \in$ $\mathcal{C}_{1}^{*}(\Gamma)$, i.e. the variational problem of minimizing $\mathcal{E}^{H}$ within a given boundary value class

$$
\begin{aligned}
& {\left[\left.X\right|_{\partial B}\right]:=\left\{Y \in H^{1,2}\left(B, \mathbb{R}^{3}\right) \cap L^{\infty}\left(B, \mathbb{R}^{3}\right) \mid Y-X \in \stackrel{H}{H}^{1,2}\left(B, \mathbb{R}^{3}\right),\|Y\|_{L^{\infty}(B)} \leqslant 1\right\}:} \\
& \wp_{H}\left(\left.X\right|_{\partial B}\right): \mathcal{E}^{H} \rightarrow \text { Min. in }\left[\left.X\right|_{\partial B}\right] .
\end{aligned}
$$

By [11], Theorem 3.6 resp. 3.7, we have the following existence, uniqueness and regularity result:

Theorem 2.1. For any $X \in \mathcal{C}_{1}^{*}(\Gamma)$ and $H \in\left(-\frac{1}{2}, \frac{1}{2}\right)$ there exists a unique solution $X^{*}:=X^{*}(X, H) \in\left[\left.X\right|_{\partial B}\right]$ of the problem $\wp_{H}\left(\left.X\right|_{\partial B}\right)$ which additionally belongs to $C^{0}\left(\bar{B}, \mathbb{R}^{3}\right) \cap C^{2}\left(B, \mathbb{R}^{3}\right)$ and solves Eq. (3) in the classical sense.

Remark 2.1. Since the unique solution $X^{*}$ of the problem $\wp_{H}\left(\left.X\right|_{\partial B}\right)$ belongs to $\left[\left.X\right|_{\partial B}\right]^{\prime}:=\left[\left.X\right|_{\partial B}\right] \cap C^{0}\left(\bar{B}, \mathbb{R}^{3}\right)$ we infer $\mathcal{E}^{H}\left(X^{*}\right)=\inf _{\left[\left.X\right|_{\partial B}\right]} \mathcal{E}^{H} \leqslant \inf _{\left[\left.X\right|_{\partial B}\right]^{]^{\prime}}} \mathcal{E}^{H} \leqslant \mathcal{E}^{H}\left(X^{*}\right)$, hence $\inf _{\left[\left.X\right|_{\partial B}\right]} \mathcal{E}^{H}=\inf _{\left[\left.X\right|_{\partial B}\right]^{\prime}} \mathcal{E}^{H}$.

Let $h(r)$ denote the uniquely determined harmonic extension $h \in C^{0}\left(\bar{B}, \mathbb{R}^{n}\right) \cap C^{2}\left(B, \mathbb{R}^{n}\right)$ of prescribed continuous boundary values $r \in C^{0}\left(\partial B, \mathbb{R}^{n}\right)$ with $\|r\|_{C^{0}(\partial B)} \leqslant 1$, for $n \geqslant 1$. From [9], Hilfssatz 5, we have the following boundary estimate for "small" solutions of (3):

Lemma 2.3. Let $|H|<\frac{1}{2}$ be arbitrarily fixed. For surfaces $X \in C^{2}\left(B, \mathbb{R}^{3}\right) \cap C^{0}\left(\bar{B}, \mathbb{R}^{3}\right)$ satisfying (3) and $\|X\|_{C^{0}(\bar{B})} \leqslant 1$ there holds

$$
\left|X\left(\rho \mathrm{e}^{\mathrm{i} \vartheta}\right)-X\left(\mathrm{e}^{\mathrm{i} \vartheta}\right)\right| \leqslant\left.\frac{|H|}{2(1-2|H|)}\left|h\left(|r|^{2}\right)\left(\rho \mathrm{e}^{\mathrm{i} \vartheta}\right)-\right| r\left(\mathrm{e}^{\mathrm{i} \vartheta}\right)\right|^{2}\left|+\frac{1-|H|}{1-2|H|}\right| h(r)\left(\rho \mathrm{e}^{\mathrm{i} \vartheta}\right)-r\left(\mathrm{e}^{\mathrm{i} \vartheta}\right) \mid
$$

for any $\rho \in[0,1]$ and $\vartheta \in[0,2 \pi]$, where $r:=\left.X\right|_{\partial B}$. 
We use this boundary estimate to prove the following central compactness result:

Theorem 2.2. A sequence $\left\{X_{k}\right\} \subset \mathcal{C}_{1}^{*}(\Gamma) \cap C^{2}\left(B, \mathbb{R}^{3}\right)$ of surfaces which solve (3) and have bounded Dirichlet integrals, i.e. $\mathcal{D}\left(X_{k}\right) \leqslant$ const., $\forall k \in \mathbb{N}$, is compact in $C^{0}\left(\bar{B}, \mathbb{R}^{3}\right)$.

Proof. On account of Hilfssatz 3 in [9] one can estimate the moduli of the gradients $\left\{\left|\nabla X_{k}\right|\right\}$ on $\overline{B_{\rho}(0)} \subset B$ uniformly by a constant $C(\rho, H)$ for any fixed $\rho \in(0,1)$, which yields

$$
\left|X_{k}\left(w_{1}\right)-X_{k}\left(w_{2}\right)\right| \leqslant C(\rho, H)\left|w_{1}-w_{2}\right| \quad \forall k \in \mathbb{N},
$$

$\forall w_{1}, w_{2} \in \overline{B_{\rho}(0)}$. Since the $X_{k}$ satisfy $\mathcal{D}\left(X_{k}\right) \leqslant$ const., $\left\|X_{k}\right\|_{C^{0}(\bar{B})} \leqslant 1$ and the three-point-condition (2) $\forall k \in \mathbb{N}$ we obtain by the Courant-Lebesgue lemma and Arzelà-Ascoli's theorem a subsequence of the boundary values $\left.X_{k}\right|_{\partial B}=: r_{k}$ (which will be renamed $\left\{r_{k}\right\}$ ) that converges in $C^{0}\left(\partial B, \mathbb{R}^{3}\right)$ to some continuous function $r$. Consequently by the weak maximum principle for harmonic functions we infer for the unique harmonic extensions $h\left(r_{k}\right)$ resp. $h\left(\left|r_{k}\right|^{2}\right)$ :

$$
\begin{aligned}
& \max _{\bar{B}}\left|h\left(\left|r_{k}\right|^{2}\right)-h\left(|r|^{2}\right)\right|=\left.\max _{\partial B}|| r_{k}\right|^{2}-|r|^{2} \mid \rightarrow 0 \quad \text { and } \\
& \max _{\bar{B}}\left|h\left(r_{k}\right)-h(r)\right| \leqslant \sqrt{3} \max _{\partial B}\left|r_{k}-r\right| \rightarrow 0,
\end{aligned}
$$

for $k \rightarrow \infty$, implying the equicontinuity of $\left\{h\left(r_{k}\right)\right\}$ and $\left\{h\left(\left|r_{k}\right|^{2}\right)\right\}$ on $\bar{B}$. Now combining this with Lemma 2.3 we obtain for a fixed $H \in\left(-\frac{1}{2}, \frac{1}{2}\right)$ : for an arbitrarily chosen $\epsilon>0$ there is a $\delta\left(\frac{\epsilon}{3}\right)>0$ such that

$$
\begin{aligned}
\left|X_{k}\left(\rho \mathrm{e}^{\mathrm{i} \vartheta}\right)-X_{k}\left(\mathrm{e}^{\mathrm{i} \vartheta}\right)\right| \leqslant & \left.\frac{|H|}{2(1-2|H|)}\left|h\left(\left|r_{k}\right|^{2}\right)\left(\rho \mathrm{e}^{\mathrm{i} \vartheta}\right)-\right| r_{k}\left(\mathrm{e}^{\mathrm{i} \vartheta}\right)\right|^{2} \mid \\
& +\frac{1-|H|}{1-2|H|}\left|h\left(r_{k}\right)\left(\rho \mathrm{e}^{\mathrm{i} \vartheta}\right)-r_{k}\left(\mathrm{e}^{\mathrm{i} \vartheta}\right)\right|<\frac{\epsilon}{3}
\end{aligned}
$$

if $1-\rho<\delta\left(\frac{\epsilon}{3}\right)$, uniformly $\forall k \in \mathbb{N}$. Together with the equicontinuity of the $X_{k}$ on every closed disc $\overline{B_{\rho}(0)} \subset B$ by (16) and the equicontinuity of the boundary values $r_{k}$ on $\partial B$ one finally achieves the equicontinuity of $\left\{X_{k}\right\}$ on $\bar{B}$ (see the proof of Theorem 2.2 in [17]). Hence by $\left\|X_{k}\right\|_{C^{0}(\bar{B})} \leqslant 1 \forall k \in \mathbb{N}$ Arzelà-Ascoli's theorem yields the assertion.

Moreover we will use the following boundary regularity result for $\mathrm{H}$-surfaces and an asymptotic expansion of the complex gradient $X_{u}-\mathrm{i} X_{v}$ about a boundary branch point due to Heinz [10], Satz 3:

Theorem 2.3. Let $X \in H^{1,2}\left(B, \mathbb{R}^{3}\right) \cap C^{0}\left(\bar{B}, \mathbb{R}^{3}\right) \cap C^{2}\left(B, \mathbb{R}^{3}\right)$ be a solution of (3) and (4) which maps an open, connected arc $\gamma \subset \partial B$ weakly monotonically into a regular, open Jordan curve $\widetilde{\Gamma}$ of class $C^{3}$.

(i) Then for every $w_{0} \in \gamma$ there is an $\epsilon_{0}>0$ such that

$$
X \in C^{1, v}\left(\bar{Z}_{w_{0}, \epsilon_{0}}, \mathbb{R}^{3}\right) \quad \forall v \in(0,1)
$$

on the closure of the "circular bigon" $Z_{w_{0}, \epsilon_{0}}:=B_{\epsilon_{0}}\left(w_{0}\right) \cap B$.

(ii) If $w_{0} \in \gamma$ is a boundary branch point of $X$ and $\left|X_{u}\right| \not \equiv 0$, then one has

$$
\left(X_{u}-\mathrm{i} X_{v}\right)(w)=a\left(w-w_{0}\right)^{k}+\mathrm{o}\left(\left|w-w_{0}\right|^{k}\right) \quad \text { for } \bar{Z}_{w_{0}, \epsilon_{0}} \ni w \rightarrow w_{0},
$$

for some complex vector $a \in \mathbb{C}^{3} \backslash\{0\}$ and a positive integer $k \in \mathbb{N}$. 
Using this theorem Hildebrandt derived in [12], Satz 3, the following

Corollary 2.1. The boundary values of an $H$-surface $X \in \mathcal{H}_{H}(\Gamma)$ perform a homeomorphism:

$$
\left.X\right|_{\partial B}: \partial B \stackrel{\cong}{\rightrightarrows} \Gamma .
$$

For surfaces $X \in H^{1,2}\left(B, \mathbb{R}^{3}\right)$ we denote its area by

$$
\mathcal{A}(X):=\int_{B}\left|X_{u} \wedge X_{v}\right| \mathrm{d} u \mathrm{~d} v .
$$

In (39) we will make use of Heinz' isoperimetric inequality for "small” H-surfaces, Theorem 3 in [8]:

Theorem 2.4. For an arbitrary "small" $H$-surface $X \in \mathcal{H}_{H}(\Gamma)$ there holds

$$
\mathcal{A}(X) \leqslant \frac{1}{4 \pi} \frac{1+h}{1-h} \mathcal{L}(\Gamma)^{2},
$$

where $h:=|H|\|X\|_{C^{0}(\bar{B})}\left(<\frac{1}{2}\right)$ and $\mathcal{L}(\Gamma):=$ length of $\Gamma$.

\section{Heinz' map $\psi$}

In this section we construct Heinz' map $\psi: T \rightarrow\left(\mathcal{C}_{1}^{*}(\Gamma),\|\cdot\|_{H^{1,2} \cap C^{0}}\right)$, where the set $T \subset \mathbb{R}^{N}$ is defined as follows:

Definition 3.1. Let $T$ be the set of $N$-tuples

$$
\left(\tau_{1}, \tau_{2}, \ldots, \tau_{N}\right)=: \tau \in(0,2 \pi)^{N} \subset \mathbb{R}^{N}
$$

which satisfy the following chain of inequalities:

$$
0=\psi_{0}<\tau_{1}<\cdots<\tau_{l}<\psi_{1}<\tau_{l+1}<\cdots<\tau_{m}<\psi_{2}<\tau_{m+1}<\cdots<\tau_{N}<2 \pi,
$$

where $l$ and $m$ are the same fixed indices as in (1).

Obviously $T$ is a convex, open and bounded subset of $\mathbb{R}^{N}$.

Definition 3.2. To each $\tau \in T$ we assign a set $\mathcal{U}(\tau)$ of surfaces $X \in \mathcal{C}_{1}^{*}(\Gamma)$ which meet the following "Courantcondition":

$$
\left.X\right|_{\partial B}\left(\mathrm{e}^{\mathrm{i} \tau_{j}}\right)=A_{j} \quad \text { for } j=1, \ldots, N .
$$

At first for any fixed $\tau \in T$ one can easily construct boundary values $r$ yielding $h(r) \in \mathcal{U}(\tau)$, hence we have

Lemma 3.1. $\mathcal{U}(\tau) \neq \emptyset \forall \tau \in T$.

As we require $\Gamma$ to be a closed polygon one easily verifies the convexity of $\mathcal{U}(\tau)$ for any $\tau \in T$, which is a rather important point.

Now we state a slight generalization of Lemma 1 in [7] which Heinz asserted without proof (see Lemma 3.2 in [17] for a proof): 
Lemma 3.2. For any two surfaces $X \in H^{1,2}\left(B, \mathbb{R}^{3}\right)$ and $Z \in C^{0}\left(\bar{B}, \mathbb{R}^{3}\right) \cap H^{1,2}\left(B, \mathbb{R}^{3}\right)$ and any monotonically increasing sequence of radii $r_{n}^{*} \nearrow 1$ there exists in each interval $\left[r_{n}^{*}-\epsilon_{n}, r_{n}^{*}+\epsilon_{n}\right]$, for $\epsilon_{n}:=\left(1-r_{n}^{*}\right) / 2$, a subset $\mathcal{R}_{n}$ with $\mathcal{L}^{1}\left(\mathcal{R}_{n}\right) \geqslant \epsilon_{n}$, such that for an arbitrary sequence of radii $\left\{\xi_{n}\right\}$ with $\xi_{n} \in \mathcal{R}_{n} \forall n \in \mathbb{N}$ there holds:

$$
\int_{0}^{2 \pi}\left|Z\left(\xi_{n} \mathrm{e}^{\mathrm{i} \varphi}\right)-Z\left(\mathrm{e}^{\mathrm{i} \varphi}\right)\right|\left|X_{\varphi}\left(\xi_{n} \mathrm{e}^{\mathrm{i} \varphi}\right)\right| \mathrm{d} \varphi \rightarrow 0 \quad \text { for } n \rightarrow \infty .
$$

The following basic integral identity, (1.9) in [7], is proved in [17], Lemma 3.3.

Lemma 3.3. For any $X, Z \in C^{0}\left(\bar{B}, \mathbb{R}^{3}\right) \cap H^{1,2}\left(B, \mathbb{R}^{3}\right)$ there holds:

$$
\begin{aligned}
& \mathcal{F}_{B_{r}(0)}(X+Z)-\mathcal{F}_{B_{r}(0)}(X) \\
& \quad=3 \int_{B_{r}(0)}\left\langle Z, X_{u} \wedge X_{v}\right\rangle \mathrm{d} u \mathrm{~d} v+\int_{B_{r}(0)}\left\langle 3 X+Z, Z_{u} \wedge Z_{v}\right\rangle \mathrm{d} u \mathrm{~d} v+\frac{1}{r} \int_{\partial B_{r}(0)}\left\langle X, Z \wedge\left(X_{\varphi}-Z_{\varphi}\right)\right\rangle \mathrm{d} s
\end{aligned}
$$

for a.e. $r \in(0,1)$.

Combining Lemma 3.2 with the identity (21) Heinz achieved in [7], Lemma 2, the following formula (see also Lemma 3.4 in [17] for a more detailed proof):

Lemma 3.4. For any two surfaces $X_{1}, X_{2} \in \mathcal{U}(\tau)$, for an arbitrary $\tau \in T$, we have:

$$
\begin{aligned}
& \mathcal{F}\left(X_{2}\right)-\mathcal{F}\left(X_{1}\right) \\
& \quad=3 \int_{B}\left\langle X_{2}-X_{1},\left(X_{1}\right)_{u} \wedge\left(X_{1}\right)_{v}\right\rangle \mathrm{d} u \mathrm{~d} v+\int_{B}\left\langle 2 X_{1}+X_{2},\left(X_{2}-X_{1}\right)_{u} \wedge\left(X_{2}-X_{1}\right)_{v}\right\rangle \mathrm{d} u \mathrm{~d} v .
\end{aligned}
$$

Recalling the convexity of the sets $\mathcal{U}(\tau)$ the above lemma yields the following crucial inequality due to Heinz [7], Lemma 3 (see also Lemma 3.5 in [17]):

Lemma 3.5. Let $\tau \in T$ be arbitrarily chosen, then for any two surfaces $X_{1}, X_{2} \in \mathcal{U}(\tau)$ there holds:

$$
\frac{1}{2}\left(\mathcal{E}^{H}\left(X_{1}\right)+\mathcal{E}^{H}\left(X_{2}\right)\right)-\mathcal{E}^{H}\left(\frac{X_{1}+X_{2}}{2}\right) \geqslant \frac{1}{2}(1-2|H|) \mathcal{D}\left(X_{1}-X_{2}\right) .
$$

Now we are prepared to prove the main result of this section using Theorems 2.1, 2.2 and the above inequality (see also Lemma 8 in [7]):

Proposition 3.1. For an arbitrary $\tau \in T$ there exists in $\mathcal{U}(\tau)$ a uniquely determined minimizer $X(\tau)$ of $\mathcal{E}^{H}$, i.e.

$$
\mathcal{E}^{H}(X(\tau))=\inf _{\mathcal{U}(\tau)} \mathcal{E}^{H}
$$

Furthermore $X(\tau)$ belongs to $C^{2}\left(B, \mathbb{R}^{3}\right)$ and solves $(3)$ in the classical sense.

Proof. Existence: Let $\left\{X_{k}\right\} \subset \mathcal{U}(\tau)$ be a minimizing sequence for $\mathcal{E}^{H}$, i.e.

$$
\mathcal{E}^{H}\left(X_{k}\right) \rightarrow \inf _{\mathcal{U}(\tau)} \mathcal{E}^{H} \text { for } k \rightarrow \infty
$$


Now Theorem 2.1 guarantees the existence of a sequence $\left\{X_{k}^{*}\right\} \subset C^{0}\left(\bar{B}, \mathbb{R}^{3}\right) \cap C^{2}\left(B, \mathbb{R}^{3}\right)$ which satisfies $X_{k}^{*} \in$ $\left[\left.X_{k}\right|_{\partial B}\right], \mathcal{E}^{H}\left(X_{k}^{*}\right)=\inf _{\left[\left.X_{k}\right|_{\partial B}\right]} \mathcal{E}^{H}$ and (3), thus $\left\{X_{k}^{*}\right\}$ is again a minimizing sequence for $\mathcal{E}^{H}$ in $\mathcal{U}(\tau)$ in particular. By (11) we see

$$
\mathcal{D}\left(X_{k}^{*}\right) \leqslant \text { const. } \forall k \in \mathbb{N},
$$

which together with $\left\|X_{k}^{*}\right\|_{C^{0}(\bar{B})} \leqslant 1$ implies $\left\|X_{k}^{*}\right\|_{H^{1,2}(B)} \leqslant$ const. $\forall k \in \mathbb{N}$. Consequently we obtain a subsequence $\left\{X_{k_{n}}^{*}\right\}$ with

$$
X_{k_{n}}^{*} \rightarrow X^{*} \quad \text { in } H^{1,2}\left(B, \mathbb{R}^{3}\right)
$$

for some $X^{*} \in H^{1,2}\left(B, \mathbb{R}^{3}\right)$, which by the theorems of Rellich and Riesz implies the existence of a further subsequence (that will be renamed $\left\{X_{k_{n}}^{*}\right\}$ ) with

$$
X_{k_{n}}^{*}(w) \rightarrow X^{*}(w) \text { for a.e. } w \in B
$$

On account of (26) Theorem 2.2 finally yields a further subsequence (that will be renamed $\left\{X_{k_{n}}^{*}\right\}$ again) satisfying

$$
X_{k_{n}}^{*} \rightarrow X^{*} \quad \text { in } C^{0}\left(\bar{B}, \mathbb{R}^{3}\right) .
$$

Hence, we obtain $X^{*} \in \mathcal{U}(\tau)$. Together with $\lim _{n \rightarrow \infty} \mathcal{E}^{H}\left(X_{k_{n}}^{*}\right)=\inf _{\mathcal{U}(\tau)} \mathcal{E}^{H}$ and the weak lower semicontinuity of $\mathcal{E}^{H}$ by Lemma 2.1 applied to (27) we finally obtain $\mathcal{E}^{H}\left(X^{*}\right)=\inf _{\mathcal{U}(\tau)} \mathcal{E}^{H}$. Furthermore together with $\left[\left.X^{*}\right|_{\partial B}\right]^{\prime}=$ $\left[\left.X^{*}\right|_{\partial B}\right] \cap C^{0}\left(\bar{B}, \mathbb{R}^{3}\right) \subset \mathcal{U}(\tau)$ and Remark 2.1 we infer:

$$
\mathcal{E}^{H}\left(X^{*}\right)=\inf _{\mathcal{U}(\tau)} \mathcal{E}^{H} \leqslant \inf _{\left[\left.X^{*}\right|_{\partial B}\right]^{\prime}} \mathcal{E}^{H}=\inf _{\left[\left.X^{*}\right|_{\partial B}\right]} \mathcal{E}^{H} \leqslant \mathcal{E}^{H}\left(X^{*}\right),
$$

thus $X^{*}$ is a solution of the Dirichlet problem $\wp_{H}\left(\left.X^{*}\right|_{\partial B}\right)$. Consequently Theorem 2.1 yields that $X^{*}$ belongs to $C^{2}\left(B, \mathbb{R}^{3}\right)$ and solves $(3)$ in the classical sense.

Uniqueness: Let $X_{1}^{*}$ and $X_{2}^{*}$ be two $\mathcal{E}^{H}$-minimizers in $\mathcal{U}(\tau)$, i.e. we have $\mathcal{E}^{H}\left(X_{1}^{*}\right)=\inf _{\mathcal{U}(\tau)} \mathcal{E}^{H}=\mathcal{E}^{H}\left(X_{2}^{*}\right)$. As $\mathcal{U}(\tau)$ is convex $\frac{1}{2}\left(X_{1}^{*}+X_{2}^{*}\right)$ belongs to $\mathcal{U}(\tau)$, thus $\mathcal{E}^{H}\left(\left(X_{1}^{*}+X_{2}^{*}\right) / 2\right) \geqslant \inf _{\mathcal{U}(\tau)} \mathcal{E}^{H}$. Consequently Lemma 3.5 yields

$$
\frac{1}{2}(1-2|H|) \mathcal{D}\left(X_{1}^{*}-X_{2}^{*}\right) \leqslant \inf _{\mathcal{U}(\tau)} \mathcal{E}^{H}-\mathcal{E}^{H}\left(\frac{X_{1}^{*}+X_{2}^{*}}{2}\right) \leqslant 0,
$$

hence $\mathcal{D}\left(X_{1}^{*}-X_{2}^{*}\right)=0$ due to $|H|<\frac{1}{2}$. Since $X_{1}^{*}$ and $X_{2}^{*}$ are continuous on $\bar{B}$ and satisfy the same three-point(and even Courant-) condition (2) we proved $X_{1}^{*} \equiv X_{2}^{*}$ on $\bar{B}$.

Now the above proposition suggests the following

Definition 3.3. For an arbitrarily chosen polygon $\Gamma \subset \mathbb{D}^{3}$ and $H \in\left(-\frac{1}{2}, \frac{1}{2}\right)$ we define "Heinz' map"

$$
\psi:=\psi^{\Gamma}(H): T \rightarrow\left(\mathcal{C}_{1}^{*}(\Gamma),\|\cdot\|_{H^{1,2} \cap C^{0}}\right) \quad \text { by } \psi(\tau):=X(\tau)
$$

and $f:=f^{\Gamma}(H): T \rightarrow \mathbb{R}$ by $f:=\mathcal{E}^{H} \circ \psi$.

\section{Proof of the reduction theorem}

On account of the invariance of the functional $\mathcal{F}$ with respect to orientation preserving diffeomorphisms $\phi: \bar{B} \cong \bar{B}$, the "positive definiteness" of $\mathcal{E}^{H}$ on $\mathcal{C}_{1}^{*}(\Gamma)(11)$, Lemmas 2.1 and 2.2, Theorem 2.2 and Proposition 3.1 the assertion (i) of the reduction theorem can be proved exactly as Theorem 6.6 in [16] (see also [7], Lemma 10).

Since orientation preserving diffeomorphisms and especially conformal automorphisms of the disc will play a central role in later sections we add a few observations on such mappings. 


\subsection{Inner variations of the disc $\bar{B}$}

Definition 4.1. A family of orientation preserving $C^{3}$-diffeomorphisms $\phi_{\epsilon}:=\phi(\cdot, \epsilon): \bar{B} \rightarrow \bar{B}, \epsilon \in\left[-\epsilon_{0}, \epsilon_{0}\right]$ (for a fixed, sufficiently small $\epsilon_{0}>0$ ), are called inner variations of $\bar{B}$ of "medium" type if they are three times continuously differentiable in $\epsilon$ on $\left[-\epsilon_{0}, \epsilon_{0}\right]$, i.e. $\phi \in C^{3}\left(\bar{B} \times\left[-\epsilon_{0}, \epsilon_{0}\right], \mathbb{R}^{2}\right)$ and a perturbation of the identity: $\phi_{0}=\mathrm{id}_{\bar{B}}$. The set of these variations of $\bar{B}$ will be denoted by $\mathcal{V}$.

Remark 4.1. (a) A family $\phi \in \mathcal{V}$ possesses a Taylor expansion with respect to $\epsilon$ : $\phi_{\epsilon}=\mathrm{id}_{\bar{B}}+\epsilon \lambda+\mathrm{o}(\epsilon)$ on $\bar{B}$, for $\epsilon \rightarrow 0$, where $\lambda:=\left.\frac{\partial}{\partial \epsilon} \phi_{\epsilon}\right|_{\epsilon=0}$ is called the generator of the family $\phi$, and its remainder of first order can be estimated by $\frac{1}{2}\|\phi\|_{C^{2}} \epsilon^{2}$.

(b) The inverse family $\left\{\phi_{\epsilon}^{-1}\right\}$ is again a family of inner variations of $\bar{B}$ of medium type and possesses the Taylor expansion $\phi_{\epsilon}^{-1}=\mathrm{id}_{\bar{B}}-\epsilon \lambda+\mathrm{o}(\epsilon)$ on $\bar{B}$, for $\epsilon \rightarrow 0$.

Remark 4.2. Now we consider the action of inner variations $\phi \in \mathcal{V}$ on the set $T$. Let $K \Subset T$ be compact and $\phi \in \mathcal{V}$ arbitrarily chosen. Then there is an $\epsilon_{0}=\epsilon_{0}(K, \phi)>0$ such that the family $\left\{\phi_{\epsilon}\right\}_{\epsilon \in\left[-\epsilon_{0}, \epsilon_{0}\right]}$ induces a flow $\left(\phi_{\epsilon}\right)_{\sharp}: K \rightarrow T$ via $\arg (\cdot): \mathbb{S}^{1} \cong \mathbb{R} /(2 \pi \mathbb{Z})$ by:

$$
\tau=\left(\tau_{1}, \ldots, \tau_{N}\right) \mapsto\left(\left.\arg \circ \phi_{\epsilon}\right|_{\partial B}\left(\mathrm{e}^{\mathrm{i} \tau_{1}}\right), \ldots,\left.\arg \circ \phi_{\epsilon}\right|_{\partial B}\left(\mathrm{e}^{\mathrm{i} \tau_{N}}\right)\right)=:\left(\phi_{\epsilon}\right)_{\sharp}(\tau) .
$$

Next we consider the map $\Lambda: \mathcal{V} \times T \rightarrow \mathbb{R}^{N}$ given by $\Lambda(\phi, \tau):=\left.\frac{\partial}{\partial \epsilon}\left(\phi_{\epsilon}\right)_{\sharp}(\tau)\right|_{\epsilon=0}$. A simple calculation yields

$$
\Lambda(\phi, \tau)=\left.\frac{\partial}{\partial \epsilon}\left(\phi_{\epsilon}\right)_{\sharp}(\tau)\right|_{\epsilon=0}=\left(\frac{1}{\mathrm{ie}^{\mathrm{i} \tau_{1}}} \lambda\left(\mathrm{e}^{\mathrm{i} \tau_{1}}\right), \ldots, \frac{1}{\mathrm{ie}^{\mathrm{i} \tau_{N}}} \lambda\left(\mathrm{e}^{\mathrm{i} \tau_{N}}\right)\right),
$$

where $\lambda$ denotes the generator of the family $\left\{\phi_{\epsilon}\right\}$. Now we fix some $\tau \in T$. For an arbitrary $\Upsilon=\left(\Upsilon_{1}, \ldots, \Upsilon_{N}\right) \in$ $\mathbb{S}^{N-1}$ we want to construct a $\phi \in \mathcal{V}$ such that the pair $(\phi, \tau)$ is mapped by $\Lambda$ onto $\Upsilon$. This can easily be carried out by means of "hill functions" $h_{m} \in C_{c}^{\infty}\left(\mathbb{R}^{2}\right)(m=1, \ldots, N)$ with respect to the fixed $\tau=\left(\tau_{1}, \ldots, \tau_{N}\right)$ which can explicitly be given by

$$
h_{m}(w):= \begin{cases}\mathrm{e}^{\frac{1}{r}} \mathrm{e}^{-1 /\left(r-\left|w-\mathrm{e}^{\mathrm{i} \tau_{m}}\right|\right)} & \text { for }\left|w-\mathrm{e}^{\mathrm{i} \tau_{m}}\right|<r, \\ 0 & \text { for }\left|w-\mathrm{e}^{\mathrm{i} \tau_{m}}\right| \geqslant r\end{cases}
$$

for $m \in\{1, \ldots, N\}$, where we have put $\tau_{N+1}:=\tau_{1}$ and require

$$
r<\frac{1}{2}\left[\min _{l \in\{1, \ldots, N\}, k \in\{0,1,2\}}\left\{\left|\mathrm{e}^{\mathrm{i} \tau_{l}}-\mathrm{e}^{\mathrm{i} \tau_{l+1}}\right|,\left|\mathrm{e}^{\mathrm{i} \tau_{l}}-\mathrm{e}^{\mathrm{i} \psi_{k}}\right|\right\}\right] .
$$

Obviously the $h_{m}$ have the following properties:

$$
\begin{aligned}
& h_{m}\left(\mathrm{e}^{\mathrm{i} \tau_{l}}\right)=\delta_{m l} \quad \text { for } m, l \in\{1, \ldots, N\}, \\
& h_{m}\left(\mathrm{e}^{\mathrm{i} \psi_{k}}\right)=0 \quad \text { for } m=1, \ldots, N \text { and } k=0,1,2 \text {. }
\end{aligned}
$$

Now we set $v:=\sum_{m=1}^{N} h_{m} \Upsilon_{m}$ on $\bar{B}$. Then the inner variations $\phi_{\epsilon}(w):=w \exp (\mathrm{i} \epsilon v(w))$, which we term $(\tau, \Upsilon)$ variations, induce

$$
\left(\phi_{\epsilon}\right)_{\sharp}(\tau)=\left(\tau_{1}+\epsilon \Upsilon_{1}, \ldots, \tau_{N}+\epsilon \Upsilon_{N}\right)=\tau+\epsilon \Upsilon \in T
$$

(for $|\epsilon|$ sufficiently small), which completes the construction.

Remark 4.3. Finally we mention that in general a family $\left\{\phi_{\epsilon}\right\} \in \mathcal{V}$ affects the three points $\left\{\mathrm{e}^{\mathrm{i} \psi_{k}}\right\}_{k=0,1,2}$; consequently the inner variations $X \circ \phi_{\epsilon}$ of some surface $X \in \mathcal{C}_{1}^{*}(\Gamma)$ might leave this class. In order to overcome this 
technical difficulty we follow Courant's idea of [2] to "renorm" $\left\{\phi_{\epsilon}\right\}$ by a family of conformal automorphisms $\left\{K_{\epsilon}\right\} \subset \operatorname{Aut}(B)$ which is uniquely determined by its desired property

$$
\tilde{\phi}_{\epsilon}\left(\mathrm{e}^{\mathrm{i} \psi_{k}}\right):=\left(\phi_{\epsilon} \circ K_{\epsilon}\right)\left(\mathrm{e}^{\mathrm{i} \psi_{k}}\right) \stackrel{!}{=} \mathrm{e}^{\mathrm{i} \psi_{k}} \quad \text { for } k=0,1,2 .
$$

Moreover via a straightforward calculation one has to ensure that this renormed family $\left\{\tilde{\phi}_{\epsilon}\right\}$ is an element of $\mathcal{V}$ again (see [15], p. 71, for a proof).

\subsection{Proof of the points (ii)-(iv) of the reduction theorem}

The proof of statement (ii) of the reduction theorem is based on the following result (see [17], Lemma 4.4, for a proof).

Proposition 4.1. Let $\left\{\phi_{\epsilon}\right\}_{\epsilon \in\left[-\epsilon_{0}, \epsilon_{0}\right]}$ be a family in $\mathcal{V}$ and $X \in H^{1,2}\left(B, \mathbb{R}^{n}\right)$ some surface, then

$$
\mathcal{D}\left(X \circ \phi_{\epsilon}\right):\left[-\epsilon_{0}, \epsilon_{0}\right] \rightarrow \mathbb{R}
$$

is twice continuously differentiable with respect to $\epsilon$ and we have (i):

$$
\left.\frac{\mathrm{d}}{\mathrm{d} \epsilon} \mathcal{D}\left(X \circ \phi_{\epsilon}\right)\right|_{\epsilon=0}=\frac{1}{2} \int_{B} a\left(\lambda_{u}^{1}-\lambda_{v}^{2}\right)+b\left(\lambda_{v}^{1}+\lambda_{u}^{2}\right) \mathrm{d} u \mathrm{~d} v=: \partial \mathcal{D}(X, \lambda),
$$

where $a:=\left|X_{u}\right|^{2}-\left|X_{v}\right|^{2}$ and $b:=2\left\langle X_{u}, X_{v}\right\rangle$ and (ii):

$$
\mathcal{E}^{H}\left(X \circ \phi_{\epsilon}\right) \leqslant \mathcal{E}^{H}(X)+2 \epsilon \partial \mathcal{D}(X, \lambda)+\epsilon^{2} c \mathcal{D}(X),
$$

where the constant $c$ depends only on $\left\{\phi_{\epsilon}\right\}$ (and $\left\{\phi_{\epsilon}^{-1}\right\}$ ) but not on $X$.

On account of the inequalities (33) and (11) the following theorem can be proved as Theorem 6.13 in [16], using the continuity of $f$, Lemma 6.12 in [16] and Remark 4.1(b) (see also Lemma 11 in [7]).

Theorem 4.1. (i) At every point $\tau \in T$ there exist all directional derivatives of $f$. (ii) Let $\tau \in T$ and $\Upsilon \in \mathbb{S}^{N-1}$ be arbitrarily chosen and consider the corresponding $(\tau, \Upsilon)$-variations $\phi_{\epsilon}(w):=\exp (\mathrm{i} \epsilon v(w))$ (see Remark 4.2), then there holds

$$
\frac{\partial}{\partial \Upsilon} f(\tau)=2 \partial \mathcal{D}(X(\tau),-\lambda),
$$

where $\lambda(w):=\mathrm{i} w v(w)$ for $w \in \bar{B}$.

Now the assertion (ii) of the reduction theorem can be proved as Theorem 6.14 in [16] combining the above theorem with Lemma 6.12 in [16] (in [7] this statement is asserted in Theorem 1 without proof).

By (11) assertion (iii) of the reduction theorem can be proved as Lemma 6.16 in [16].

Now using $f \in C^{1}(T)$ the proof of Theorem 4.1 yields the following counterpart of the statement of Theorem 4.1(ii) (see Corollary 6.15 in [16] for a proof).

Corollary 4.1. Consider some $\phi \in \mathcal{V}$ which leaves the three points $\left\{\mathrm{e}^{\mathrm{i} \psi_{k}}\right\}$ invariant, its generator $\lambda$, some fixed point $\tau \in T$ and the corresponding vector $\Upsilon:=\Lambda(\phi, \tau)=\left.\frac{\partial}{\partial \epsilon}\left(\phi_{\epsilon}\right)_{\sharp}(\tau)\right|_{\epsilon=0}$ (see Remark 4.2). Then

$$
\partial \mathcal{D}(X(\tau), \lambda)=-\frac{1}{2} \frac{\partial}{\partial \Upsilon} f(\tau)=-\frac{1}{2}\langle\nabla f(\tau), \Upsilon\rangle .
$$

A combination of this representation of inner variations of the Dirichlet integral with Remark 4.3 and Lemma 6.18, Proposition 6.19 in [16] yields analogously to Theorem 6.17 in [16] (see also Theorem 2 in [7]): 
Corollary 4.2. If $\tau \in T$ is a critical point of $f$, then $X(\tau)$ is conformally parametrized on B, i.e. $X(\tau)$ satisfies (4).

Now recalling Proposition 3.1 the above corollary just states that the restriction of $\psi$ to the set $K(f)$ of critical points of $f$ maps into $\mathcal{H}_{H}(\Gamma)$, i.e.

$$
\operatorname{im}\left(\left.\psi\right|_{K(f)}\right) \subset \mathcal{H}_{H}(\Gamma) .
$$

In order to achieve even

$$
\operatorname{im}\left(\left.\psi\right|_{K(f)}\right)=\mathcal{H}_{H}(\Gamma)
$$

one needs Lemma 6 in [7] (see also Theorem 4.3 in [17] for a more detailed proof):

Theorem 4.2. If an $H$-surface $X$ belongs to the intersection $\mathcal{H}_{H}(\Gamma) \cap \mathcal{U}(\tau)$ for some $\tau \in T$, then there holds

$$
\mathcal{E}^{H}(X)=\inf _{\mathcal{U}(\tau)} \mathcal{E}^{H}
$$

Now let there be given an arbitrary $\mathrm{H}$-surface $X \in \mathcal{H}_{H}(\Gamma)$. Then it must be contained in some class $\mathcal{U}(\tau)$ for some $\tau \in T$ due to the surjectivity of its boundary values onto $\Gamma$. Hence, combining the above theorem with Proposition 3.1 we expose $X$ to be $\psi(\tau)$, which means $\operatorname{im}(\psi) \supset \mathcal{H}_{H}(\Gamma)$. Furthermore by (32) we see $\partial \mathcal{D}(X, \lambda)=0 \forall \lambda \in C^{1}\left(\bar{B}, \mathbb{R}^{2}\right)$ since $X$ is conformally parametrized on $B$, i.e. since $X$ satisfies (4). Consequently by Theorem 4.1(ii) $\tau$ must be a critical point of $f$, which implies even $\operatorname{im}\left(\left.\psi\right|_{K(f)}\right) \supset \mathcal{H}_{H}(\Gamma)$. Thus together with (36) we finally obtain (37). Furthermore Corollary 2.1 implies

\section{Corollary 4.3.}

$$
\left.\psi\right|_{K(f)}: K(f) \longmapsto \mathcal{H}_{H}(\Gamma) \quad \text { is injective. }
$$

Proof. If we assume the contrary, i.e. that there exist points $\left\{\tau^{1} \neq \tau^{2}\right\} \subset K(f)$ with $X\left(\tau^{1}\right)=\psi\left(\tau^{1}\right)=\psi\left(\tau^{2}\right)=$ $X\left(\tau^{2}\right)$, then especially the boundary values $\left.X\left(\tau^{1}\right)\right|_{\partial B}=\left.X\left(\tau^{2}\right)\right|_{\partial B} \in C^{0}\left(\partial B, \mathbb{R}^{3}\right)$ would have to coincide. However in the tuples $\left(\tau_{1}^{1}, \ldots, \tau_{N}^{1}\right)=\tau^{1} \neq \tau^{2}=\left(\tau_{1}^{2}, \ldots, \tau_{N}^{2}\right)$ there is at least one different pair $\tau_{j}^{1} \neq \tau_{j}^{2}\left(\right.$ e.g. $\left.\tau_{j}^{1}<\tau_{j}^{2}\right)$, and due to the weak monotonicity of the boundary values $\left.X\left(\tau^{1}\right)\right|_{\partial B}=\left.X\left(\tau^{2}\right)\right|_{\partial B}$ these would have to be constant on the open, connected arc between $\exp \left(\mathrm{i} \tau_{j}^{1}\right)$ and $\exp \left(\mathrm{i} \tau_{j}^{2}\right)$, in contradiction to Corollary 2.1 .

Moreover combining $\mathcal{A}(X)=\mathcal{D}(X)$ for any $X \in \mathcal{H}_{H}(\Gamma)$ with (37), (11) and Heinz' isoperimetric inequality (18) we obtain for every critical point $\tau \in K(f)$ the estimate

$$
0 \leqslant f(\tau)=\mathcal{E}^{H}(\psi(\tau))<\text { const. }(|H|, \mathcal{L}(\Gamma))=: c .
$$

Together with point (iii) of the reduction theorem this yields

$$
K(f) \Subset f^{-1}([0, c)) \Subset T .
$$

Hence, $K(f)$ reveals to be a compact subset of $T$, which together with the continuity of $\psi$, (37) and Corollary 4.3 implies assertion (iv) of the reduction theorem.

\section{Application of Dold's fixed point theory and Čech cohomology}

In this section we combine the reduction theorem with singular homology, Čech cohomology [6] and Dold's fixed point theory [4] in order to define the H-surface-index $\mathcal{I}: \mathbf{K}_{H}(\Gamma) \rightarrow \mathbb{Z}$ and derive its asserted properties. 


\subsection{Definition of the $H$-surface index $\mathcal{I}$}

We define a continuous flow $\phi: T \times\left[-t_{0}, t_{0}\right] \rightarrow T$ by

$$
\phi(\tau, t):=\tau-t \eta(\tau) \nabla f(\tau)
$$

for a sufficiently small $t_{0}>0$, depending on $\nabla f$ and a cut-off function $\eta \in C_{c}^{0}(T,[0,1])$ to be defined precisely in Definition 5.1 below with the property

$$
f^{-1}([0, c]) \Subset[\eta=1],
$$

where $c=c(|H|, \mathcal{L}(\Gamma))$ is the constant from (39). We compute

$$
\frac{\mathrm{d}}{\mathrm{d} t} f(\phi(\tau, t))=-\eta(\tau)\langle\nabla f(\phi(\tau, t)), \nabla f(\tau)\rangle
$$

$\forall t \in\left[-t_{0}, t_{0}\right]$ and $\forall \tau \in T$. At first we state the following elementary

Lemma 5.1. If there holds $|\nabla f| \geqslant \delta$ for some $\delta>0$ on a compact subset $K \subset T$, then there exists some $t^{\sharp}=$ $t^{\sharp}(K, \nabla f) \in\left(0, t_{0}\right]$ such that

$$
f(\phi(\tau, t)) \leqslant f(\tau)-\frac{t}{2} \eta(\tau) \delta^{2} \leqslant f(\tau)
$$

uniformly $\forall \tau \in K$ and $\forall t \in\left[0, t^{\sharp}\right]$ and

$$
f(\phi(\tau, t)) \geqslant f(\tau)+\frac{|t|}{2} \eta(\tau) \delta^{2} \geqslant f(\tau)
$$

uniformly $\forall \tau \in K$ and $\forall t \in\left[-t^{\sharp}, 0\right]$.

Proof. From $|\nabla f| \geqslant \delta>0$ on $K$, the compactness of $K$, the continuity of $\nabla f$ and (43) one can easily derive the existence of some $t^{\sharp} \in\left(0, t_{0}\right]$ (depending on $K$ and $\nabla f$ ) such that

$$
\frac{\mathrm{d}}{\mathrm{d} t} f(\phi(\tau, t)) \leqslant-\frac{\eta(\tau)}{2}|\nabla f(\tau)|^{2}
$$

uniformly $\forall \tau \in K$ and $\forall t \in\left[-t^{\sharp}, t^{\sharp}\right]$. Hence, by the fundamental theorem of calculus we obtain:

$$
f(\phi(\tau, t))-f(\tau)=\int_{0}^{t} \frac{\mathrm{d}}{\mathrm{d} t^{\prime}} f\left(\phi\left(\tau, t^{\prime}\right)\right) \mathrm{d} t^{\prime} \leqslant-t \frac{\eta(\tau)}{2}|\nabla f(\tau)|^{2} \leqslant-t \frac{\eta(\tau)}{2} \delta^{2}
$$

$\forall \tau \in K$ and $\forall t \in\left[0, t^{\sharp}\right]$ and

$$
f(\phi(\tau, t))-f(\tau)=\int_{0}^{t} \frac{\mathrm{d}}{\mathrm{d} t^{\prime}} f\left(\phi\left(\tau, t^{\prime}\right)\right) \mathrm{d} t^{\prime}=-\int_{t}^{0} \frac{\mathrm{d}}{\mathrm{d} t^{\prime}} f\left(\phi\left(\tau, t^{\prime}\right)\right) \mathrm{d} t^{\prime} \geqslant|t| \frac{\eta(\tau)}{2}|\nabla f(\tau)|^{2} \geqslant|t| \frac{\eta(\tau)}{2} \delta^{2}
$$

$\forall \tau \in K$ and $\forall t \in\left[-t^{\sharp}, 0\right]$.

From the above inequalities we derive

Corollary 5.1. There is some $t^{*} \in\left(0, t_{0}\right]$ such that

$$
\begin{aligned}
& \left.\phi(\cdot, t)\right|_{f^{-1}([0, c])}: f^{-1}([0, c]) \rightarrow f^{-1}([0, c]) \quad \text { and } \\
& \left.\phi(\cdot, t)\right|_{f^{-1}([0, c])} \simeq \operatorname{id}_{f^{-1}([0, c])} \quad \operatorname{rel} K(f)
\end{aligned}
$$


for an arbitrary $t \in\left[0, t^{*}\right]$ and analogously

$$
\begin{aligned}
& \left.\phi(\cdot, t)\right|_{f^{-1}((c, \infty))}: f^{-1}((c, \infty)) \rightarrow f^{-1}((c, \infty)) \quad \text { and } \\
& \left.\phi(\cdot, t)\right|_{f^{-1}((c, \infty))} \simeq \operatorname{id}_{f^{-1}((c, \infty))} \quad \operatorname{rel}[\eta=0]
\end{aligned}
$$

for an arbitrary $t \in\left[-t^{*}, 0\right]$.

Proof. Due to (40) there exists some open set $O$ such that $K(f) \Subset O \Subset f^{-1}([0, c])$. On the compact set $f^{-1}([0, c]) \backslash O$ we thus have $|\nabla f| \geqslant \delta_{1}$ for some $\delta_{1}>0$ and Lemma 5.1 yields the existence of some $t_{1}^{\sharp} \in\left(0, t_{0}\right]$ such that $\operatorname{im}\left(\left.\phi(\cdot, t)\right|_{f^{-1}([0, c]) \backslash O}\right) \subset f^{-1}([0, c]) \forall t \in\left[0, t_{1}^{\sharp}\right]$. Furthermore due to the compactness of $\bar{O} \Subset f^{-1}([0, c])$ and the continuity of $\nabla f$ there is some $t_{2}^{\sharp} \in\left(0, t_{0}\right]$ such that $\operatorname{im}(\phi(\cdot, t) \mid \bar{O}) \subset f^{-1}([0, c])$ $\forall t \in\left[0, t_{2}^{\sharp}\right]$. Hence, the assertion (46) holds for $t^{\sharp}:=\min \left\{t_{1}^{\sharp}, t_{2}^{\sharp}\right\}$ and we may simply use a linear homotopy. Analogously we argue that by (40) there holds $|\nabla f| \geqslant \delta_{2}$ for some $\delta_{2}>0$ on the compact set $f^{-1}([c, \infty) \cap \operatorname{supp}(\eta)$. Thus by Lemma 5.1 there is some $t_{3}^{\sharp} \in\left(0, t_{0}\right] \operatorname{such}$ that $\operatorname{im}\left(\left.\phi(\cdot, t)\right|_{f^{-1}((c, \infty)) \cap \operatorname{supp}(\eta)}\right) \subset f^{-1}((c, \infty)) \forall t \in\left[-t_{3}^{\sharp}, 0\right]$. On $[\eta=0]$ the assertion (47) holds trivially $\forall t \in\left[-t_{0}, t_{0}\right]$. Thus also in (47) we may use a linear homotopy and the corollary is proved for $t^{*}:=\min \left\{t^{\sharp}, t_{3}^{\sharp}\right\}$.

Thus by (40), (42) and (46) we obtain the following identity:

$$
K(f)=\operatorname{Fix}\left(\left.\phi(\cdot, t)\right|_{f^{-1}([0, c])}\right)=: F
$$

for any $t \in\left(0, t^{*}\right]$, where Fix denotes the fixed point set of a continuous selfmap.

Now we compactify the convex open set $T \cong \mathbb{D}^{N}$ by some homeomorphism

$$
\varphi: \bar{T} \backslash \partial T \stackrel{\cong}{\rightrightarrows} \mathbb{S}^{N} \backslash\{\infty\},
$$

where $\infty$ can be chosen as the "North Pole" $e_{N+1}:=(0, \ldots, 0,1) \in \mathbb{R}^{N+1}$, and we transport the flow $\phi$ onto the $\mathbb{S}^{N}$ by setting

$$
\tilde{\phi}(\cdot, t):=\varphi \circ \phi(\cdot, t) \circ \varphi^{-1}: \mathbb{S}^{N} \backslash\{\infty\} \rightarrow \mathbb{S}^{N} \backslash\{\infty\}
$$

$\forall t \in\left[-t_{0}, t_{0}\right]$. Due to $\operatorname{supp}(\eta) \Subset T$ there holds $\tilde{\phi}(\cdot, t) \equiv$ id on some punctured neighborhood about $\infty$ on the $\mathbb{S}^{N} \forall|t| \leqslant t_{0}$. Hence, $\tilde{\phi}(\infty, t):=\infty \forall t \in\left[-t_{0}, t_{0}\right]$ exposes to be the unique continuous extension of $\tilde{\phi}$ onto the entire $\mathbb{S}^{N}$. Moreover due to point (iii) of the reduction theorem we may extend

$$
\tilde{f}:=f \circ \varphi^{-1}: \mathbb{S}^{N} \backslash\{\infty\} \rightarrow \mathbb{R}
$$

to a continuous function $\tilde{f}: \mathbb{S}^{N} \rightarrow \mathbb{R} \cup\{\infty\}$ by setting $\tilde{f}(\infty):=\infty$. In particular (47) transforms to

$$
\begin{aligned}
& \left.\tilde{\phi}(\cdot, t)\right|_{\tilde{f}^{-1}((c, \infty])}: \tilde{f}^{-1}((c, \infty]) \rightarrow \tilde{f}^{-1}((c, \infty]) \quad \text { and } \\
& \left.\tilde{\phi}(\cdot, t)\right|_{\tilde{f}^{-1}((c, \infty])} \simeq \operatorname{id}_{\tilde{f}^{-1}((c, \infty])} \quad \operatorname{rel} \varphi([\eta=0]) \cup\{\infty\}
\end{aligned}
$$

for an arbitrary $t \in\left[-t^{*}, 0\right]$.

Now we choose some sufficiently small contractible neighborhood $V \cong \mathbb{D}^{N}$ of the "North Pole" $\infty$ in $\mathbb{S}^{N}$ which satisfies $V \Subset \tilde{f}^{-1}([c, \infty])$. Moreover we define

$$
M:=\max \left\{\tilde{f}(\tau) \mid \tau \in \tilde{f}^{-1}([c, \infty]) \backslash V\right\}
$$

and see firstly due to $\left(\tilde{f}^{-1}([c, \infty]) \backslash \stackrel{\circ}{V}\right) \neq \emptyset$ and (40) $M>c$ and secondly

$$
\tilde{f}^{-1}((M, \infty]) \subset V
$$

just by construction of $M$, for if there were a point $\tau \in \tilde{f}^{-1}((M, \infty]) \backslash V$, then by (51) we would have $\tilde{f}(\tau) \leqslant M$, in contradiction to $\tau \in \tilde{f}^{-1}((M, \infty])$.

Now we are prepared for 
Definition 5.1. We define the cut-off function $\eta \in C_{c}^{0}(T,[0,1])$ in (41) by

$$
\begin{aligned}
& \eta(\tau):=\frac{\operatorname{dist}\left(\tau, f^{-1}([M+1, \infty))\right)}{\operatorname{dist}\left(\tau, f^{-1}([M+1, \infty))\right)+\operatorname{dist}\left(\tau, f^{-1}([0, M])\right)}, \quad \tau \in T, \\
& \text { thus } \quad[\eta=1]=f^{-1}([0, M]) \text { and } \quad[\eta=0]=f^{-1}([M+1, \infty)) .
\end{aligned}
$$

Due to $M>c \eta$ satisfies the requirement (42). Moreover by this precise definition of $\eta$ we implicitly fix an interval $\left[-t_{0}, t_{0}\right]$ for the "maximal duration" of the flow $\phi$ which consequently only depends on $f, \nabla f$ and $\mathcal{L}(\Gamma)$.

Now let $K \subset T$ be a compact subset and $U \Subset T$ some open neighborhood of $K$ in $T$, then there correspond via $\varphi$

$$
K \subset U \Subset T \stackrel{\varphi}{\longleftrightarrow} \widetilde{K} \subset \widetilde{U} \Subset \mathbb{S}^{N} \backslash\{\infty\} .
$$

In the sequel $H_{*}\left({ }_{-}\right):=H_{*}\left({ }_{-}, \mathbb{Z}\right)$ will denote the functor of singular homology with coefficients in $\mathbb{Z}$.

Definition 5.2. Let $o$ be a fixed generator of $H_{N}\left(\mathbb{S}^{N}\right) \cong \mathbb{Z}$. With respect to $o$ we define the fundamental class $o_{K}$ around $K$ (see [5], p. 202) as the image of $o$ under the following composition:

$$
\begin{aligned}
& H_{N}\left(\mathbb{S}^{N}\right) \stackrel{i_{*}}{\rightarrow} H_{N}\left(\mathbb{S}^{N}, \mathbb{S}^{N} \backslash \widetilde{K}\right) \stackrel{\cong}{\rightarrow} H_{N}(\widetilde{U}, \widetilde{U} \backslash \widetilde{K}) \stackrel{\cong}{\rightarrow} H_{N}(U, U \backslash K), \\
& o \mapsto i_{*}(o) \mapsto o_{K},
\end{aligned}
$$

where we used the induced homomorphism $i_{*}$ of the inclusion $i:\left(\mathbb{S}^{N}, \emptyset\right) \hookrightarrow\left(\mathbb{S}^{N}, \mathbb{S}^{N} \backslash \widetilde{K}\right)$, the excisionisomorphism and $\varphi_{*}$.

Remark 5.1. We note that for two open neighborhoods $U_{1} \subset U_{2}$ of $K$ the induced homomorphism $i_{*}$ of the inclusion $i:\left(U_{1}, U_{1} \backslash K\right) \hookrightarrow\left(U_{2}, U_{2} \backslash K\right)$ takes $o_{K}^{1}$ into $o_{K}^{2}$, hence, we are allowed to drop $U$ in the notation of $o_{K}$.

Now we fix some $t \in\left(0, t^{*}\right]$ and abbreviate $\phi:=\phi(\cdot, t)$.

Definition 5.3. A compact subset $P$ of $F$ is termed a part of $F$ if it possesses an open neighborhood $U$ in $T$ with $P=F \cap U$.

By (40), (42) and (48) we can choose $U \Subset[\eta=1]$. Now we consider the following map of pairs:

$$
\operatorname{id}_{U}-\left.\phi\right|_{U}=\left.t \nabla f\right|_{U}:(U, U \backslash P) \rightarrow\left(\mathbb{R}^{N}, \mathbb{R}^{N} \backslash\{0\}\right) .
$$

Following Dold ([5], p. 203) application of $H_{*}$ yields

Definition 5.4. We define the fixed point index of $\phi$ around a part $P \subset F$ by

$$
\begin{aligned}
& \left(\left.t \nabla f\right|_{U}\right)_{*}: H_{N}(U, U \backslash P) \rightarrow H_{N}\left(\mathbb{R}^{N}, \mathbb{R}^{N} \backslash\{0\}\right) \cong \mathbb{Z} \\
& o_{P} \mapsto \underline{I_{\left.\phi\right|_{U}}} \cdot o_{0} .
\end{aligned}
$$

Remark 5.2. We note that on account of Remark 5.1 the above definition does not depend on $U$, since for two neighborhoods $U_{1} \stackrel{i}{\hookrightarrow} U_{2}$ of $P$ we derive from $i_{*}\left(o_{P}^{1}\right)=o_{P}^{2}$ :

$$
I_{\left.\phi\right|_{U_{1}}} \cdot o_{0}=\left(\left.t \nabla f\right|_{U_{1}}\right)_{*}\left(o_{P}^{1}\right)=\left(\left.t \nabla f\right|_{U_{2}}\right)_{*} \circ i_{*}\left(o_{P}^{1}\right)=\left(\left.t \nabla f\right|_{U_{2}}\right)_{*}\left(o_{P}^{2}\right)=I_{\left.\phi\right|_{U_{2}}} \cdot o_{0} .
$$

Furthermore the homotopy invariance of singular homology guarantees the independence of $I_{\left.\phi\right|_{U}}$ from the chosen $t \in\left(0, t^{*}\right]$. 
Remark 5.3. In Proposition 5.10 of [5], p. 205, the definition of the fixed point index of the set of continuous functions $g: U \rightarrow \mathbb{R}^{M}$, that are defined on open sets $U$ of $\mathbb{R}^{M}(M \geqslant 1)$, is uniquely extended to maps which are defined on open subsets $U$ of an arbitrary topological space $Y, g: U \rightarrow Y$, where $U$ additionally has to be a ENR:=Euclidean Neighborhood Retract; i.e. there has to exist an open subset $V$ of $\mathbb{R}^{M}$, an embedding $i: U \hookrightarrow V$ and a continuous map $r: V \rightarrow U$ (retraction) such that $r \circ i=\mathrm{id}_{U}$. Now if $g: U \rightarrow Y$ is a continuous map with compact fixed point set Dold defines its generalized fixed point index by

$$
\left.I_{g}:=I_{i \circ\left(\left.g \circ r\right|_{(g \circ r)}-1(U)\right.}\right) \cdot
$$

In Proposition 5.10 of [5], p. 205, Dold shows that this extension of the fixed point index does not depend on the choices of $i$ and $r$ and that all properties of the "special" index remain valid (see [5], p. 206). This generalization will be valuable for us when we will consider some neighborhood $U \Subset[\eta=1]$ of a part $P \subset F$ with $P=F \cap U$, which is a compact ENR and on which $\phi$ acts as a selfmap (thus here is $Y:=U$ ), and when we will need the unique existence of $I_{\left.\phi\right|_{U}}$ and its coincidence with the fixed point index as defined in Definition 5.4:

$$
I_{\left.\phi\right|_{U}}=I_{\left.\phi\right|_{W}}
$$

for some open neighborhood $W$ of $P$ with $W \subset U$ (see [5], p. 206, (5.11)).

Proposition 5.1. The parts of $F$ and those of $\mathcal{H}_{H}(\Gamma)$ correspond to each other via $\psi$ :

$$
F \supset P \stackrel{\psi}{\longleftrightarrow} \mathcal{P} \subset \mathcal{H}_{H}(\Gamma)
$$

Proof. (i) Let $P$ be a (nonempty) part of $F$, then $\psi(P)$ is compact, thus especially closed in $\left(\mathcal{C}_{1}^{*}(\Gamma),\|\cdot\|_{C^{0} \cap H^{1,2}}\right)$. Now we suppose that $\psi(P)$ would not be a part of $\mathcal{H}_{H}(\Gamma)$. Then there would have to exist a sequence $\left\{X_{i}\right\} \subset$ $\mathcal{H}_{H}(\Gamma) \backslash \psi(P)$ with

$$
\operatorname{dist}\left(X_{i}, \psi(P)\right) \rightarrow 0 \quad \text { for } i \rightarrow \infty .
$$

By the compactness of $F(6)$ yields the compactness of $\mathcal{H}_{H}(\Gamma)$. Thus there would be a subsequence $X_{i_{k}}$ converging to some $X \in \mathcal{H}_{H}(\Gamma)$ and (55) implies $X \in \overline{\psi(P)}=\psi(P)$. Hence, again by (6) we would obtain:

$$
F \backslash P \ni \psi^{-1}\left(X_{i_{k}}\right) \rightarrow \psi^{-1}(X) \in P \quad \text { for } k \rightarrow \infty,
$$

which is impossible since the part $P$ is separated from $F \backslash P$ by some open neighborhood.

(ii) Now let $\mathcal{P}$ be a part of $\mathcal{H}_{H}(\Gamma)$, i.e. there exists an open neighborhood $O \subset \mathcal{C}_{1}^{*}(\Gamma)$ of $\mathcal{P}$ with $O \cap \mathcal{H}_{H}(\Gamma)=\mathcal{P}$. By (6) $\psi^{-1}(\mathcal{P})$ is compact and due to the continuity of $\psi$ on $T \psi^{-1}(O)$ is an open neighborhood of $\psi^{-1}(\mathcal{P})$ in $T$. Now by $(O \backslash \mathcal{P}) \cap \mathcal{H}_{H}(\Gamma)=\emptyset$ one easily concludes $\left(\psi^{-1}(O) \backslash \psi^{-1}(\mathcal{P})\right) \cap F=\emptyset$, which means that $\psi^{-1}(O)$ separates $\psi^{-1}(\mathcal{P})$ from $F \backslash \psi^{-1}(\mathcal{P})$, i.e. that $\psi^{-1}(\mathcal{P})$ is a part.

Now the above correspondence, Definition 5.4 and Remark 5.2 suggest

Definition 5.5 (Definition of the H-surface index). We define the H-surface index

$$
\mathcal{I}: \mathbf{K}_{H}(\Gamma) \rightarrow \mathbb{Z} \quad \text { by } \mathcal{I}(\mathcal{P}):=I_{\left.\phi\right|_{U}},
$$

where $U$ is some open neighborhood of the part $P:=\psi^{-1}(\mathcal{P})$ in $T$ satisfying $P=U \cap F$ and $U \Subset[\eta=1]$.

From the additivity of Dold's fixed point index $I$ (see [5], p. 203 and p. 206) we can immediately derive the additivity of the H-surface index $\mathcal{I}$, just as asserted in (8). Furthermore we note $\mathcal{I}(\emptyset)=0$. 


\subsection{Proof of the point (i) of the main theorem}

Due to (40) we may apply Lemma 5.1 to $K:=f^{-1}([c, M+1])$, where $M$ was defined in (51), and obtain the inequality (45) with some appropriate $t^{\sharp} \in\left(0, t_{0}\right]$ and $\delta>0$. Iteration of (45) yields

Lemma 5.2. For $k \in \mathbb{N}$ with $k>\frac{M-c}{\epsilon}, \epsilon:=\frac{t^{\sharp}}{2} \delta^{2}$, there holds:

$$
f\left(\phi^{k}\left(\tau,-t^{\sharp}\right)\right)>M \quad \forall \tau \in f^{-1}((c, \infty)),
$$

where $\left.\phi^{k}\left(\cdot,-t^{\sharp}\right)\right|_{f^{-1}((c, \infty))}$ denotes the kth iterate of the selfmap $\left.\phi\left(\cdot,-t^{\sharp}\right)\right|_{f^{-1}((c, \infty))}($ see $(47))$.

Proof. We abbreviate $\phi^{\sharp}:=\left.\phi\left(\cdot,-t^{\sharp}\right)\right|_{f^{-1}((c, \infty))}$. For some arbitrarily chosen $\tau \in f^{-1}((c, \infty))$ we distinguish the following cases:

$$
\text { 1) } \left.\tau \in f^{-1}((c, M]), \quad \text { 2) } \tau \in f^{-1}((M, M+1]), \quad 3\right) \tau \in f^{-1}((M+1, \infty)) .
$$

For a $\tau$ satisfying case 3 the assertion is an immediate consequence of $[\eta=0]=f^{-1}([M+1, \infty))$. If case 2 holds true for $\tau$, then the assertion follows from (45) on account of the possible choice $K:=f^{-1}([c, M+1])$ in Lemma 5.1. In case 1 we apply $\phi^{\sharp}$ to this $\tau$ and classify $\phi^{\sharp}(\tau)$ with respect to the cases $1,2,3$ and so on. Now it is impossible to obtain $k$ consecutive times case 1 for $k>\frac{M-c}{\epsilon}$, for if we again choose $K:=f^{-1}([c, M+1])$ in Lemma 5.1 and note $[\eta=1]=f^{-1}([0, M])$, then an application of (45) to each iterate $\left(\phi^{\sharp}\right)^{j}(\tau) \in f^{-1}((c, M])$, $j=0, \ldots, k-1$, finally implies:

$$
f\left(\left(\phi^{\sharp}\right)^{k}(\tau)\right) \geqslant f(\tau)+k \epsilon>c+M-c=M,
$$

with $\epsilon:=\frac{t^{\sharp}}{2} \delta^{2}$.

Transferred onto the $\mathbb{S}^{N}$ this means together with (52):

$$
\left.\tilde{\phi}^{k}\left(\cdot,-t^{\sharp}\right)\right|_{\tilde{f}^{-1}((c, \infty])}: \tilde{f}^{-1}((c, \infty]) \rightarrow \tilde{f}^{-1}((M, \infty]) \subset V .
$$

Now we consider the reduced homology ladder of the pair $\left(\tilde{f}^{-1}((c, \infty]), V\right)$ and the map $\tilde{\phi}^{k}:=$ $\left.\tilde{\phi}^{k}\left(\cdot,-t^{\sharp}\right)\right|_{\tilde{f}^{-1}((c, \infty])}($ with coefficients in $\mathbb{Z})$ :

$$
\begin{aligned}
& \widetilde{H}_{m}(V) \stackrel{i_{*}}{\longrightarrow} \widetilde{H}_{m}\left(\tilde{f}^{-1}((c, \infty])\right) \stackrel{\cong}{\longrightarrow} H_{m}\left(\tilde{f}^{-1}((c, \infty]), V\right) \stackrel{\partial_{*}}{\longrightarrow} \widetilde{H}_{m-1}(V)
\end{aligned}
$$

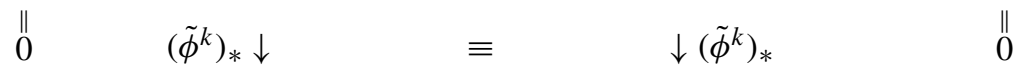

$$
\begin{aligned}
& \widetilde{H}_{m}(V) \stackrel{i_{*}}{\longrightarrow} \widetilde{H}_{m}\left(\tilde{f}^{-1}((c, \infty])\right) \stackrel{\cong}{\longrightarrow} H_{m}\left(\tilde{f}^{-1}((c, \infty]), V\right) \stackrel{\partial_{*}}{\longrightarrow} \widetilde{H}_{m-1}(V)
\end{aligned}
$$

$\forall m \in \mathbb{Z}$. Firstly due to $V \cong \mathbb{D}^{N}$ there holds $\widetilde{H}_{*}(V)=0$, exposing the two middle horizontal arrows to be isomorphisms, secondly by (50) we obtain for the left vertical arrow $\left(\tilde{\phi}^{k}\right)_{*}=\operatorname{id}_{\widetilde{H}_{m}\left(\tilde{f}^{-1}((c, \infty])\right)}$, and thirdly by $(57)$ there holds for the right vertical arrow $\operatorname{im}\left(\tilde{\phi}^{k}\right)_{*}=0 \in H_{m}\left(\tilde{f}^{-1}((c, \infty]), V\right) \forall m \in \mathbb{Z}$. Using the commutativity of the diagram we gain $\operatorname{im}_{(i d} \widetilde{H}_{m}\left(\tilde{f}^{-1}((c, \infty])\right)=0$, hence, together with the universal coefficient formula of singular homology (see [5], p. 153) in particular:

$$
\widetilde{H}_{m}\left(\tilde{f}^{-1}((c, \infty]), \mathbb{Q}\right)=0 \quad \forall m \in \mathbb{Z} .
$$

Since $c$ is a regular value of $f, f^{-1}([0, c])$ turns out to be a compact manifold with boundary on account of the inverse function theorem (see [13], Section 4.1). In particular, $f^{-1}([0, c])$ is locally compact and locally contractible and consequently a compact ENR on account of a theorem due to Borsuk (see [5], p. 83). Thus by 
$\tilde{f}^{-1}((c, \infty])=\mathbb{S}^{N} \backslash \tilde{f}^{-1}([0, c])$ we derive from (58) and Alexander duality (with coefficients in $\mathbb{Q}$ ) (see [5], p. 301):

$$
\widetilde{H}^{N-i}\left(\tilde{f}^{-1}([0, c]), \mathbb{Q}\right) \cong \widetilde{H}_{i-1}\left(\tilde{f}^{-1}((c, \infty]), \mathbb{Q}\right)=0 \quad \forall i \in \mathbb{Z} .
$$

Moreover the universal coefficient formula of singular cohomology yields (see Satz 13.4.8 in [18]): $0=$ $\widetilde{H}^{N-i}\left(\tilde{f}^{-1}([0, c]), \mathbb{Q}\right) \cong \operatorname{Hom}_{\mathbb{Q}}\left(\widetilde{H}_{N-i}\left(\tilde{f}^{-1}([0, c]), \mathbb{Q}\right), \mathbb{Q}\right)$. Hence, we achieve

$$
\widetilde{H}_{N-i}\left(f^{-1}([0, c]), \mathbb{Q}\right)=0 \quad \forall i \in \mathbb{Z},
$$

which implies for the Euler-characteristic:

$$
\chi\left(f^{-1}([0, c])\right):=\chi\left(f^{-1}([0, c]), \mathbb{Q}\right):=\sum_{j \in \mathbb{Z}}(-1)^{j} \operatorname{dim}_{\mathbb{Q}} H_{j}\left(f^{-1}([0, c]), \mathbb{Q}\right)=1 .
$$

Now let us consider sequences $V:=\left\{V_{j}\right\}_{j \in \mathbb{Z}}$ of finite dimensional vector spaces over $\mathbb{Q}$. We define the Lefschetz number $\Lambda$ of a sequence $(\beta: V \rightarrow V):=\left\{\beta_{j}: V_{j} \rightarrow V_{j}\right\}_{j \in \mathbb{Z}}$ of endomorphisms with $\operatorname{im}\left(\beta_{j}\right) \neq\{0\}$ for only finitely many $j \in \mathbb{Z}$ by $\Lambda(\beta):=\sum_{j \in \mathbb{Z}}(-1)^{j} \operatorname{Spur}\left(\beta_{j}\right)$ (compare with [5], p. 208, for a more general definition).

We abbreviate $\left.\phi\right|_{f^{-1}([0, c])}:=\left.\phi(\cdot, t)\right|_{f^{-1}([0, c])}$ for some arbitrary $t \in\left(0, t^{*}\right]$. By (46) $\left.\phi\right|_{f^{-1}([0, c])}$ yields a selfmap of the compact ENR $f^{-1}([0, c])$ and is homotopic to $\operatorname{id}_{f^{-1}([0, c])}$, thus the Lefschetz number $\Lambda\left(\left(\left.\phi\right|_{f^{-1}([0, c])}\right)_{*}\right)$ reduces to the Euler-characteristic $\chi\left(f^{-1}([0, c])\right)$. Moreover since $\mathcal{H}_{H}(\Gamma)$ corresponds to $F \Subset f^{-1}([0, c))$ via $\psi$ we infer from the definition of $\mathcal{I}$, (54) with $W:=f^{-1}([0, c))$ and $U:=f^{-1}([0, c])$, Dold's fixed point theorem (see [5], p. 209 and p. 212) and (59):

$$
\mathcal{I}\left(\mathcal{H}_{H}(\Gamma)\right)=I_{\left.\phi\right|_{f^{-1}([0, c))}}=I_{\left.\phi\right|_{f^{-1}([0, c])}}=\Lambda\left(\left(\left.\phi\right|_{f^{-1}([0, c])}\right)_{*}\right)=\chi\left(f^{-1}([0, c])\right)=1 .
$$

Recalling $\mathcal{I}(\emptyset)=0$ we verify $\mathcal{H}_{H}(\Gamma) \neq \emptyset$ as asserted in Corollary (i) of Section 1.

\subsection{Proof of point (iii) of the main theorem}

At first it should be pointed out that we are going to use two different constructions of Čech cohomology in this subsection (see Section 5.2 in [17]). The more general one is the Čech-extension $\check{H}$ of singular cohomology $H^{*}$ from the category $\mathcal{P O} \mathcal{L}_{\mathcal{K}}$ of compact polyhedrons and simplicial maps to the category $\mathcal{T} \mathcal{O P} \mathcal{K}$ of compact Hausdorff spaces and continuous maps, following Eilenberg and Steenrod [6], Chapters IX-X, resp. Dold [5], pp. 348-366. For compact subsets $K \subset \mathbb{R}^{M}(M \geqslant 1)$ we can also define its Čech cohomology groups by the direct limit $\lim _{\rightarrow}\left\{H^{*}(V)\right\}_{V \in \Theta_{M}(K)}$, where $\Theta_{M}(K)$ denotes the set of all neighborhoods $V$ of $K$ in $\mathbb{R}^{M}$ which is directed by "inverse inclusion", i.e. $V^{\prime} \geqslant V: \Leftrightarrow V^{\prime} \subseteq V$ for $V, V^{\prime} \in \Theta_{M}(K)$ (see [5], p. 281). Now by the continuity of $\check{H}$ on $\mathcal{T} \mathcal{O} \mathcal{P}_{\mathcal{K}}$ (see [6], p. 261) these two notions of Čech cohomology groups fortunately coincide (up to isomorphism) on compact subsets $K$ of $\mathbb{R}^{M}$ (see [17], p. 82). Hence, together with the homotopy invariance of $\check{H}$ on $\mathcal{T} \mathcal{O} \mathcal{P}_{\mathcal{K}}$ (see [5], p. 363) and its functor properties we achieve

Proposition 5.2. Let $\mathcal{P}$ be an arbitrary (nonempty) part of $\mathcal{H}_{H}(\Gamma)$ and $P$ the corresponding part of $K(f)$ via $\psi$, then there holds:

$$
\left.\check{\psi}\right|_{P}: \check{H}(\mathcal{P}) \stackrel{\cong}{\rightrightarrows} \check{H}(P) \cong \lim _{\rightarrow}\left\{H^{*}(V)\right\}_{V \in \Theta_{N}(P)},
$$

for coefficients in $\mathbb{Z}$ or $\mathbb{Q}$.

Now we are prepared for the proof of point (iii) of the main theorem.

(a) We consider an $\mathcal{E}^{H}$-minimizing part $\mathcal{P}(\neq \emptyset)$ of $\mathcal{H}_{H}(\Gamma)$, i.e. there hold (1) and (2) of Definition 1.1(ii) for some neighborhood $\mathcal{U}$ of $\mathcal{P}$ in $\mathcal{C}_{1}^{*}(\Gamma)$ with $\mathcal{U} \cap \mathcal{H}_{H}(\Gamma)=\mathcal{P}$. Proposition 5.1 yields that $P:=\psi^{-1}(\mathcal{P})$ is a part of $K(f)$ and that $U \cap K(f)=P$ for $U:=\psi^{-1}(\mathcal{U})$. Consequently we obtain for $f=\mathcal{E}^{H} \circ \psi$ : 


$$
\begin{array}{ll}
f(\tau) \equiv b & \forall \tau \in P \quad \text { and } \\
f(\tau)>b & \forall \tau \in U \backslash P .
\end{array}
$$

(62) is verified easily since (2) of Definition 1.1(ii) immediately implies $f(\cdot) \geqslant b$ on $U$. Hence, supposing the existence of a point $\tau \in U \backslash P$ with $f(\tau)=b$, by the openness of $U \tau$ would have to be a local minimizer of $f$, in particular a critical point of $f$ in contradiction to $U \cap K(f)=P$.

As $U$ is an open neighborhood of the compact set $P(\neq \emptyset)$ we have $\epsilon:=\operatorname{dist}(\partial U, P)>0$. We define the $\sigma$ neighborhood of $P$ by $P^{\sigma}:=\{\tau \in U \mid \operatorname{dist}(\tau, P)<\sigma\}$, for $\sigma \in(0, \epsilon]$, and choose open neighborhoods $V_{0}, V_{1}$ of $P$ with $V_{1} \Subset V_{0} \Subset P^{\epsilon} \subset U$. Moreover we set

$$
a_{1}:=\min \left\{f(\tau) \mid \tau \in \overline{V_{0} \backslash V_{1}}\right\} .
$$

On account of $\overline{V_{0} \backslash V_{1}} \subset U \backslash P$ and (62) we see $a_{1}>b$, which together with (61) and $U \cap K(f)=P$ implies that $a_{1}$ is a regular value of $\left.f\right|_{U}$. Furthermore we define

$$
M_{a_{1}}:=\left\{\tau \in V_{0} \mid f(\tau) \leqslant a_{1}\right\}
$$

and obtain

Lemma 5.3. (i) ${\stackrel{\circ}{a_{1}}}_{a_{1}}=\left(\left.f\right|_{V_{0}}\right)^{-1}\left(\left[b, a_{1}\right)\right)$, (ii) $\stackrel{\circ}{M}_{a_{1}}$ is an open neighborhood of $P$, (iii) ${\stackrel{\circ}{a_{1}}}_{\subset} \subset V_{1}$, (iv) $M_{a_{1}}$ is $a$ compact manifold with boundary, especially a compact ENR.

Proof. (i) $\stackrel{\circ}{M}_{a_{1}} \supseteq\left(\left.f\right|_{V_{0}}\right)^{-1}\left(\left[b, a_{1}\right)\right)$ is obvious, for the definition of $M_{a_{1}},(61)$, (62) and the continuity of $f$ imply that $\left(\left.f\right|_{V_{0}}\right)^{-1}\left(\left[b, a_{1}\right)\right)$ is an open subset of $M_{a_{1}}$, hence must be contained in the open kernel ${\stackrel{\circ}{a_{1}}}_{1}$. In particular, we notice that $\stackrel{\circ}{M}_{a_{1}}$ is not empty. Now we suppose that ${\stackrel{\circ}{a_{1}}} \subseteq\left(\left.f\right|_{V_{0}}\right)^{-1}\left(\left[b, a_{1}\right)\right)$ would be wrong, then there would exist a point $\tau \in{\stackrel{\circ}{a_{1}}}_{1}$ with $f(\tau)=a_{1}$, which by (64) would expose to be a local maximizer of $\left.f\right|_{U}$, thus especially a critical point of $\left.f\right|_{U}$, contradicting the fact that $a_{1}$ is a regular value of $\left.f\right|_{U}$. Now assertion (ii) follows immediately from (i), $a_{1}>b$ and $P=\left(\left.f\right|_{V_{0}}\right)^{-1}(b)$. For (iii) we argue similarly as in (52). For a point $\tau \in \stackrel{\circ}{M}_{a_{1}} \backslash V_{1}(63)$ and (64) imply $f(\tau) \geqslant a_{1}$, in contradiction to $\tau \in\left(\left.f\right|_{V_{0}}\right)^{-1}\left(\left[b, a_{1}\right)\right)$ by (i). In particular, this implies $M_{a_{1}} \subset \bar{V}_{1} \Subset V_{0}$, which guarantees the compactness of $M_{a_{1}}$.

Now since $a_{1}$ is a regular value of $\left.f\right|_{U}$ assertion (iv) follows from the inverse function theorem (see [13], Section 4.1) together with Borsuk's theorem (see [5], p. 83).

Since in the above lemma we exposed $M_{a_{1}}$ to be a neighborhood of $P$ we can again choose an open neighborhood $V_{2}$ of $P$ with $V_{2} \Subset M_{a_{1}} \cap P^{\epsilon / 2}\left(\subset \bar{V}_{1}\right)$ and define $a_{2}:=\min _{\overline{V_{1} \backslash V_{2}}} f$. By the above reasoning we see $a_{2}>b$, thus $a_{2}$ is a regular value of $\left.f\right|_{U}$. Consequently we obtain as in the proof of Lemma 5.3 that

$$
M_{a_{2}}:=\left\{\tau \in V_{1} \mid f(\tau) \leqslant a_{2}\right\}
$$

is a compact ENR, $\stackrel{\circ}{M}_{a_{2}} \subset V_{2}$ and that $\stackrel{\circ}{M}_{a_{2}}=\left(\left.f\right|_{V_{1}}\right)^{-1}\left(\left[b, a_{2}\right)\right)$ is an open neighborhood of $P=\left(\left.f\right|_{V_{1}}\right)^{-1}(b)$. Hence, inductively we obtain

Corollary 5.2. (i) There exist sequences $\left\{V_{n}\right\}_{n \in \mathbb{N}}$ resp. $\left\{M_{a_{n}}\right\}_{n \in \mathbb{N}}$ consisting of open sets resp. compact ENRs which are cofinal in $\Theta_{N}(P)$ and satisfy

$$
M_{a_{n}} \subset \bar{V}_{n} \Subset M_{a_{n-1}} \cap P^{\epsilon / n} \quad \forall n \in \mathbb{N}
$$

(with $M_{a_{0}}:=\emptyset$ ).

(ii) $\left\{a_{n}\right\}_{n \in \mathbb{N}}$ is a monotonically decreasing sequence with $a_{n} \searrow b$.

Proof. (i) follows by induction over $n \in \mathbb{N}$, where Lemma 5.3 yields the start of the induction (with $M_{a_{0}}:=\emptyset$ ) and the step $n \mapsto n+1$ works just like the step $1 \mapsto 2$ by choosing an open neighborhood $V_{n}$ of $P$ with $V_{n} \Subset$ $M_{a_{n-1}} \cap P^{\epsilon / n}$ and defining $a_{n}:=\min _{\overline{V_{n-1 \backslash V}}} f$ and then $M_{a_{n}}:=\left(\left.f\right|_{V_{n-1}}\right)^{-1}\left(\left[b, a_{n}\right]\right)$. 
(ii) From the above induction we immediately obtain $a_{n}>b \forall n \in \mathbb{N}$. Now we suppose that $a_{n}>a_{n+1}$ would be wrong for some $n \in \mathbb{N}$. Combining the points (i) and (iii) of Lemma 5.3 (in step $n$ ) we would obtain that $\dot{M}_{a_{n}}=$ $\left(\left.f\right|_{V_{n-1}}\right)^{-1}\left(\left[b, a_{n}\right)\right)=\left(\left.f\right|_{V_{n}}\right)^{-1}\left(\left[b, a_{n}\right)\right)$, which together with ${\stackrel{\circ}{a_{n+1}}}_{a_{n}}=\left(\left.f\right|_{V_{n}}\right)^{-1}\left(\left[b, a_{n+1}\right)\right)$ and our hypothesis, $a_{n} \leqslant a_{n+1}$, would imply:

$$
\stackrel{\circ}{M}_{a_{n}}=\left(\left.f\right|_{V_{n}}\right)^{-1}\left(\left[b, a_{n}\right)\right) \subset\left(\left.f\right|_{V_{n}}\right)^{-1}\left(\left[b, a_{n+1}\right)\right)=\stackrel{\circ}{M}_{a_{n+1}},
$$

in contradiction to $\stackrel{\circ}{M}_{a_{n}} \ni \stackrel{\circ}{M}_{a_{n+1}}$ by (65). Now let $\tau^{n} \in \overline{V_{n-1} \backslash V_{n}}$, for $n \in \mathbb{N}$, be an arbitrarily chosen sequence with $f\left(\tau^{n}\right)=a_{n}$. By (65) there holds $\tau^{n} \in P^{\epsilon /(n-1)} \forall n \in \mathbb{N}$. Combining this with (61) and the uniform continuity of $f$ on $\bar{V}_{0}$ we finally achieve: $a_{n}=f\left(\tau^{n}\right) \searrow b$.

Now we consider some consecutive pair $M_{a_{n}} \ni M_{a_{n+1}}\left(\ni \bar{V}_{n+2}\right)$. From $U \cap K(f)=P$ and the continuity of $\nabla f$ we derive the existence of some $\delta_{n}>0$ such that $|\nabla f| \geqslant \delta_{n}$ on $M_{a_{n}} \backslash V_{n+2}$. Hence, we can apply Lemma 5.1 with $K:=M_{a_{n}} \backslash V_{n+2}$ and obtain inequality (44) for some $t_{n}^{\sharp} \in\left(0, t_{0}\right]$ :

$$
f(\phi(\tau, t)) \leqslant f(\tau)-\frac{t}{2} \delta_{n}^{2} \leqslant f(\tau) \quad \forall \tau \in M_{a_{n}} \backslash V_{n+2}
$$

and $\forall t \in\left[0, t_{n}^{\sharp}\right]$, where we note $\eta \equiv 1$ on $M_{a_{n}}$ due to (40) and (42) having chosen $n$ sufficiently large. Analogously to (46) we obtain

Lemma 5.4. There exists some $\hat{t}_{n} \in\left(0, t_{n}^{\sharp}\right]$ such that

$$
\begin{aligned}
& \left.\phi(\cdot, t)\right|_{M_{a_{n}}}: M_{a_{n}} \rightarrow M_{a_{n}},\left.\quad \phi(\cdot, t)\right|_{M_{a_{n}}} \simeq \operatorname{id}_{M_{a_{n}}} \quad \text { rel } P \quad \text { and } \\
& \left.\phi(\cdot, t)\right|_{a_{a_{n+1}}}: M_{a_{n+1}} \rightarrow M_{a_{n+1}},\left.\quad \phi(\cdot, t)\right|_{M_{a_{n+1}}} \simeq \operatorname{id}_{M_{a_{n+1}}} \quad \text { rel } P
\end{aligned}
$$

for some arbitrary $t \in\left[0, \hat{t}_{n}\right]$.

Proof. By $M_{a_{n}} \subset \bar{V}_{n} \ni V_{n-1}$, the compactness of $M_{a_{n}}$ and the continuity of $\nabla f$, there exists some $\tilde{t}_{n}^{1} \in\left(0, t_{n}^{\sharp}\right]$ such that

$$
\operatorname{im}\left(\left.\phi(\cdot, t)\right|_{M_{a_{n}}}\right) \subset V_{n-1} \quad \forall t \in\left[0, \tilde{t}_{n}^{1}\right] .
$$

Analogously one gains the existence of a $\tilde{t}_{n}^{2} \in\left(0, t_{n}^{\sharp}\right]$ with $\operatorname{im}\left(\left.\phi(\cdot, t)\right|_{M_{a_{n+1}}}\right) \subset V_{n} \forall t \in\left[0, \tilde{t}_{n}^{2}\right]$. We set $\tilde{t}_{n}:=$ $\min \left\{\tilde{t}_{n}^{1}, \tilde{t}_{n}^{2}\right\}$. Now displacing $f^{-1}([0, c])$ by $M_{a_{n}}$ (resp. $\left.M_{a_{n+1}}\right), O$ by $V_{n+2}$ and $K(f)$ by $P$ in the proof of statement (46) one achieves the following reasoning: Combination of (66) and (69) yields

$$
\phi(\tau, t) \in\left(\left.f\right|_{V_{n-1}}\right)^{-1}\left(\left[b, a_{n}\right]\right)=M_{a_{n}} \quad \forall \tau \in M_{a_{n}} \backslash V_{n+2}
$$

and $\forall t \in\left[0, \tilde{t}_{n}\right]$. Moreover by the compactness of $\bar{V}_{n+2} \Subset M_{a_{n}}$ and the continuity of $\nabla f$ there exists a $\hat{t}_{n}^{1} \in\left(0, \tilde{t}_{n}\right]$ such that $\phi(\tau, t) \in M_{a_{n}} \forall \tau \in \bar{V}_{n+2}$ and $\forall t \in\left[0, \hat{t}_{n}^{1}\right]$ still holds, which proves the first statement of (67) on [0, $\left.\hat{t}_{n}^{1}\right]$. We thus can use a linear homotopy in (67). By the construction of $\tilde{t}_{n}$ and $\bar{V}_{n+2} \Subset M_{a_{n+1}}$ one analogously obtains the existence of some $\hat{t}_{n}^{2} \in\left(0, \tilde{t}_{n}\right]$ such that both statements of (68) hold on $\left[0, \hat{t}_{n}^{2}\right]$. Hence, the lemma is proved for $\hat{t}_{n}:=\min \left\{\hat{t}_{n}^{1}, \hat{t}_{n}^{2}\right\}$.

Similarly to the proof of Lemma 5.2 we now obtain

Lemma 5.5. For $k_{n} \in \mathbb{N}$ with $k_{n}>\left(a_{n}-a_{n+1}\right) / \rho_{n}, \rho_{n}:=\left(\hat{t}_{n} / 2\right) \delta_{n}^{2}$, there holds:

$$
\left.\phi^{k_{n}}\right|_{M_{a_{n}}}: M_{a_{n}} \rightarrow M_{a_{n+1}},
$$

where we $\operatorname{set} \phi:=\phi\left(\cdot, \hat{t}_{n}\right)$. 
Proof. We distinguish again the cases: 1) $\tau \in M_{a_{n+1}}$, 2) $\tau \in M_{a_{n}} \backslash M_{a_{n+1}}$.

In case $1(68)$ immediately yields $\phi^{k_{n}}(\tau) \in M_{a_{n+1}}$. In case 2 we see by (67) that $\phi(\tau) \in M_{a_{n}}$, thus we can again classify $\phi(\tau)$ with respect to the cases 1 and 2 and so on. Now it is impossible to obtain $k_{n}$ consecutive times case 2, for due to $M_{a_{n}} \backslash M_{a_{n+1}} \subset M_{a_{n}} \backslash V_{n+2}$ an application of (66) to each iterate $\phi^{j}(\tau) \in M_{a_{n}} \backslash M_{a_{n+1}}, j=0, \ldots, k_{n}-1$, finally yields:

$$
f\left(\phi^{k_{n}}(\tau)\right) \leqslant f(\tau)-k_{n} \rho_{n}<a_{n}-\left(a_{n}-a_{n+1}\right)=a_{n+1} .
$$

Thus due to $a_{n+1}<a_{n}$ we have especially $f\left(\phi^{k_{n}}(\tau)\right)<a_{n}$, and by (67) we know that $\phi^{k_{n}}(\tau) \in M_{a_{n}}=$ $\left(\left.f\right|_{V_{n-1}}\right)^{-1}\left(\left[b, a_{n}\right]\right)$. Hence, we conclude $\phi^{k_{n}}(\tau) \in\left(\left.f\right|_{V_{n-1}}\right)^{-1}\left(\left[b, a_{n}\right)\right)=\stackrel{\circ}{M}_{a_{n}} \subset V_{n}$. Combining this with (71) we finally achieve $\phi^{k_{n}}(\tau) \in\left(\left.f\right|_{V_{n}}\right)^{-1}\left(\left[b, a_{n+1}\right)\right)=\stackrel{\circ}{M}_{a_{n+1}} \subset M_{a_{n+1}}$.

Similarly to $\star$ in Subsection 5.2 we now use the cohomology ladder of the pair $\left(M_{a_{n}}, M_{a_{n+1}}\right)$ and the map $\phi^{k_{n}}:=\phi^{k_{n}}\left(\cdot, \hat{t}_{n}\right):\left(M_{a_{n}}, M_{a_{n+1}}\right) \rightarrow\left(M_{a_{n}}, M_{a_{n+1}}\right)$ with coefficients in $\mathbb{Z}$ resp. $\mathbb{Q}$ (see [5], p. 151):

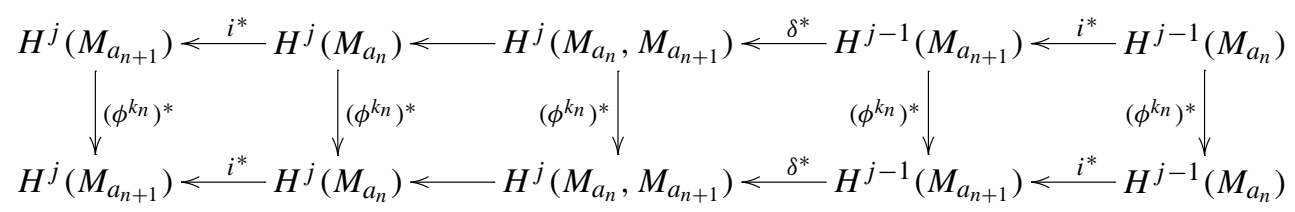

$\forall j \in \mathbb{Z}$, where $i^{*}$ is induced by the inclusion $i=i_{n}^{n+1}: M_{a_{n+1}} \hookrightarrow M_{a_{n}}$. On account of the homotopies in (67) and (68) we obtain $\left(\left.\phi^{k_{n}}\right|_{M_{a_{n+1}}}\right)^{*}=\mathrm{id}_{H^{*}\left(M_{a_{n+1}}\right)}$ and $\left(\left.\phi^{k_{n}}\right|_{M_{a_{n}}}\right)^{*}=\mathrm{id}_{H^{*}\left(M_{a_{n}}\right)}$ for the four exterior vertical arrows and by (70) $\operatorname{im}\left(\phi^{k_{n}}\right)^{*}=0 \in H^{*}\left(M_{a_{n}}, M_{a_{n+1}}\right)$ for the middle vertical arrow. On the other hand we see together with the exactness and commutativity of the cohomology ladder directly or by the five lemma $H^{*}\left(M_{a_{n}}, M_{a_{n+1}}\right)=0$, hence

$$
i^{*}=\left(i_{n}^{n+1}\right)^{*}: H^{*}\left(M_{a_{n}}\right) \stackrel{\cong}{\rightarrow} H^{*}\left(M_{a_{n+1}}\right)
$$

for an arbitrary (sufficiently large chosen) $n \in \mathbb{N}$, thus without loss of generality $\forall n \in \mathbb{N}$. Now we consider the direct system $\left\{H^{j}\left(M_{a_{n}}\right)\right\}_{n \in \mathbb{N}}$, whose morphisms are given by compositions of the isomorphisms

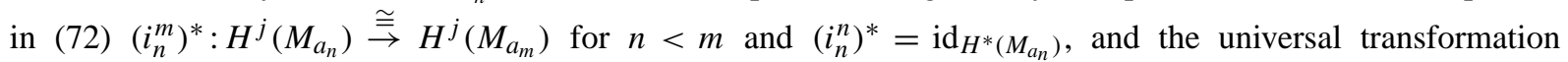
$u:=\left\{u_{n}\right\}:\left\{H^{*}\left(M_{a_{n}}\right)\right\} \rightarrow \lim _{\rightarrow} H^{*}\left(M_{a_{m}}\right)$ (see [5], p. 272-274). Applying Proposition 5.18 in [5], p. 277, to $u$ we easily obtain

$$
u_{n}: H^{*}\left(M_{a_{n}}\right) \stackrel{\cong}{\rightarrow} \lim _{\rightarrow} H^{*}\left(M_{a_{m}}\right) \quad \forall n \in \mathbb{N},
$$

and since $\left\{M_{a_{n}}\right\}_{n \in \mathbb{N}}$ is a cofinal sequence in $\Theta_{N}(P)$ by Corollary 5.2 we conclude together with (60) (see Proposition 5.17 in [5], p. 276):

$$
\check{H}^{j}(\mathcal{P}) \cong \check{H}^{j}(P) \cong H^{j}\left(M_{a_{n}}\right) \quad \forall n \in \mathbb{N},
$$

for an arbitrary $j \in \mathbb{Z}$ and coefficients in $\mathbb{Z}$ or $\mathbb{Q}$. Now we consider again the compactification $\varphi: \bar{T} \backslash \partial T \stackrel{\widetilde{\widetilde{P}}}{\cong}$ $\mathbb{S}^{N} \backslash\{\infty\}$ from $(49)$ and set $\widetilde{P}:=\varphi(P)$. For a point $\widetilde{Q} \in \widetilde{P}$ we denote by $i: \widetilde{Q} \hookrightarrow \widetilde{P}$ the inclusion and by $r: \widetilde{P} \rightarrow \widetilde{Q}$ the retraction, then we have $\operatorname{id}_{\breve{H}(\widetilde{Q})}=(r \circ \breve{i})=\breve{i} \circ \check{r}$. Hence, the long exact Čech cohomology sequence (see [6], p. 245) yields for every $j \geqslant N>0$ :

$$
\cdots 0=\check{H}^{j}(\widetilde{Q}) \stackrel{\check{i}}{\longleftarrow} \check{H}^{j}(\widetilde{P}) \cong \check{H}^{j}(\widetilde{P}, \widetilde{Q}) \stackrel{\check{\delta}=0}{\longleftarrow} \check{H}^{j-1}(\widetilde{Q}) \stackrel{\check{i}}{\longleftarrow} \cdots .
$$

Combination with (60) and Alexander duality (see [5], p. 301) finally yields:

$$
\check{H}^{j}(\mathcal{P}) \cong \check{H}^{j}(\widetilde{P}, \widetilde{Q}) \cong \widetilde{H}_{N-j-1}\left(\mathbb{S}^{N} \backslash \widetilde{P}\right)=0 \quad \text { for } j \geqslant N>0
$$


and coefficients in $\mathbb{Z}$ or $\mathbb{Q}$. With regard to the proof of formula (9) below in (c) it is already noted here that Corollary 6.5 in [5], p. 73, implies

$$
H_{j}\left(\tilde{M}_{a_{n}}, \mathbb{Z}\right)=0 \text { for } j \geqslant N
$$

since $\widetilde{M}_{a_{n}}=\varphi\left(M_{a_{n}}\right) \ni \mathbb{S}^{N} \backslash\{\infty\}$ is a neighborhood retract in $\mathbb{S}^{N}$. Furthermore Proposition 4.11 in [5], p. 103, guarantees that $H_{j}\left(M_{a_{n}}, \mathbb{Z}\right)$ is finitely generated $\forall j \in \mathbb{Z}$ because $M_{a_{n}}$ is a compact ENR. Together with the universal coefficient formulas of singular (co-) homology (see [5], p. 153) we obtain $\forall j \in \mathbb{Z}$ :

$$
\begin{aligned}
& H^{j}\left(M_{a_{n}}, \mathbb{Z}\right) \cong \mathbb{Z}^{r_{j}} \oplus \operatorname{Tor}\left(H_{j-1}\left(M_{a_{n}}, \mathbb{Z}\right)\right) \text { and } \\
& H_{j}\left(M_{a_{n}}, \mathbb{Q}\right) \cong H_{j}\left(M_{a_{n}}, \mathbb{Z}\right) \otimes \mathbb{Q} \oplus \operatorname{Tor}\left(H_{j-1}\left(M_{a_{n}}, \mathbb{Z}\right), \mathbb{Q}\right) \cong \mathbb{Q}^{r_{j}},
\end{aligned}
$$

with $r_{j}:=\operatorname{rang}\left(H_{j}\left(M_{a_{n}}, \mathbb{Z}\right)\right)<\infty, \operatorname{since} \operatorname{Ext}\left(\mathbb{Z}^{r_{j-1}}, \mathbb{Z}\right)$ vanishes and $\mathbb{Q}$ is torsion-free. Hence, by (74) and (78) we conclude that $\check{H}^{j}(\mathcal{P}, \mathbb{Z})$ is finitely generated $\forall j \in \mathbb{Z}$ and non-trivial at most in degrees $j=0, \ldots, N-1$ due to (76). Moreover combining (74), Satz 13.4.8 in [18] and (79) we obtain

$$
\check{H}^{j}(\mathcal{P}, \mathbb{Q}) \cong H^{j}\left(M_{a_{n}}, \mathbb{Q}\right) \cong\left(H_{j}\left(M_{a_{n}}, \mathbb{Q}\right)\right)^{*} \cong \mathbb{Q}^{r_{j}} \quad \forall j \in \mathbb{Z}
$$

with $r_{j} \neq 0$ at most for $j=0, \ldots, N-1$ on account of (76) (or (77)).

(b) Since $\widetilde{P}=\varphi(P)$ is compact $\mathbb{S}^{N} \backslash \widetilde{P}$ must be open in $\mathbb{S}^{N}$, thus especially a neighborhood retract in $\mathbb{S}^{N}$. Now for an arbitrary subset $A \subset \mathbb{S}^{N}$ we denote by $\Gamma(A)$ the additive group of locally constant functions $\alpha: A \rightarrow$ $\widetilde{H}_{N}\left(\mathbb{S}^{N}\right)$ and by $Z(A)$ the number of connected components of $A$. A combination of Lemma 7.1 in [5], p. 78, with the $J$-isomorphism (for the $\mathbb{S}^{N}$ ) from Proposition 6.4(b) in [5], p. 72, applied to $\mathbb{S}^{N} \backslash \widetilde{P} \subset \mathbb{S}^{N}$, i.e.

$$
J: H_{N}\left(\mathbb{S}^{N}, \mathbb{S}^{N} \backslash \widetilde{P}\right) \stackrel{\cong}{\rightarrow} \Gamma\left(\mathbb{S}^{N} \backslash\left(\mathbb{S}^{N} \backslash \widetilde{P}\right)\right)=\Gamma(\widetilde{P})
$$

(with coefficients in $\mathbb{Z}$ ), yields the following formula:

$$
Z(\widetilde{P})=\operatorname{rang}(\Gamma(\widetilde{P}))=\operatorname{rang}\left(H_{N}\left(\mathbb{S}^{N}, \mathbb{S}^{N} \backslash \widetilde{P}\right)\right) .
$$

Now we apply Lefschetz duality (see Proposition 7.2 in [5], p. 292) to the compact subset $\widetilde{P}$ of $\mathbb{S}^{N}$, i.e.

$$
-\frown o_{\widetilde{P}}: \check{H}^{0}(\widetilde{P}) \cong H_{N}\left(\mathbb{S}^{N}, \mathbb{S}^{N} \backslash \widetilde{P}\right)
$$

(with coefficients in $\mathbb{Z}$ ), and achieve together with (81), (74) and (78)

$$
Z(\mathcal{P})=Z(\widetilde{P})=\operatorname{rang}\left(\check{H}^{0}(\widetilde{P})\right)=\operatorname{rang}\left(H^{0}\left(M_{a_{n}}, \mathbb{Z}\right)\right)=r_{0}<\infty
$$

for the number of connected components of $\mathcal{P}$.

Remark 5.4. We shall note that the finiteness of the number of connected components of an arbitrary part $\mathcal{P}$ does not follow a priori from its compactness since it is not known if $\mathcal{P}$ is locally connected.

(c) Furthermore we can proceed as in Subsection 5.2. We abbreviate $\left.\phi\right|_{(-)}:=\left.\phi\left(\cdot, \hat{t}_{n}\right)\right|_{(-)}$for the $\hat{t}_{n}>0$ determined in Lemma 5.4. By (67) $\left.\phi\right|_{M_{a_{n}}}$ yields a selfmap of the compact ENR $M_{a_{n}}$. Since $\mathcal{P}$ corresponds to $P$ via $\psi$ and since $V_{n+1}$ is an open neighborhood of $P$ with $V_{n+1} \cap F=P$ and $V_{n+1} \ni M_{a_{n}}$ we can derive from the definition of $\mathcal{I}$, (54) with $W:=V_{n+1}$ and $U:=M_{a_{n}}$, Dold's fixed point theorem (see [5], p. 209 resp. p. 212), the homotopy in (67), (79), (77) and (80):

$$
\mathcal{I}(\mathcal{P})=I_{\left.\phi\right|_{V_{n+1}}}=I_{\phi_{\left.\right|_{a_{n}}}}=\Lambda\left(\left(\left.\phi\right|_{M_{a_{n}}}\right)_{*}\right)=\chi\left(M_{a_{n}}, \mathbb{Q}\right)=\sum_{j=0}^{N-1}(-1)^{j} r_{j}=\check{\chi}(\mathcal{P}, \mathbb{Q}) .
$$

Now combining the points (i), (ii) and (iii) of the main theorem we immediately obtain Corollary (ii) of Section 1. 


\section{References}

[1] R. Böhme, A.J. Tromba, The index theorem for classical minimal surfaces, Ann. of Math. 113 (2) (1981) 447-499.

[2] R. Courant, Critical points and unstable minimal surfaces, Proc. N.A.S. 27 (1941) 51-57.

[3] R. Courant, On the first variation of the Dirichlet-Douglas integral and on the method of gradients, Proc. N.A.S. 27 (1941) $242-248$.

[4] A. Dold, Fixed point index and fixed point theorem for Euclidean neighborhood retracts, Topology 4 (1965) 1-8.

[5] A. Dold, Lectures on Algebraic Topology, second ed., Grundlehren Math. Wiss., vol. 200, Springer-Verlag, 1980.

[6] S. Eilenberg, N. Steenrod, Foundations of Algebraic Topology, Princeton University Press, 1952.

[7] E. Heinz, On surfaces of constant mean curvature with polygonal boundaries, Arch. Rational Mech. Anal. 36 (1970) $335-347$.

[8] E. Heinz, An inequality of isoperimetric type for surfaces of constant mean curvature, Arch. Rational Mech. Anal. 33 (1970) $155-168$.

[9] E. Heinz, Über die Existenz einer Fläche konstanter mittlerer Krümmung bei vorgegebener Berandung, Math. Ann. 127 (1954) $258-287$.

[10] E. Heinz, Ein Regularitätssatz für Flächen beschränkter mittlerer Krümmung, Nachr. Akad. Wiss. Göttingen Math.-Phys. K1. II. 12 (1969) $107-118$

[11] S. Hildebrandt, Über Flächen konstanter mittlerer Krümmung, Math. Z. 112 (1969) 107-144.

[12] S. Hildebrandt, Randwertprobleme für Flächen mit vorgeschriebener mittlerer Krümmung und Anwendungen auf die Kapillaritätstheorie, Math. Z. 112 (1969) 205-213.

[13] S. Hildebrandt, Analysis 2, Springer, 2003.

[14] C. Imbusch, Eine Anwendung des Mountain-Pass-Lemmas auf den Fragenkreis des Plateauschen Problems und eine Alternative zur DreiPunkte-Bedingung, Diplomarbeit, Bonn, 1997.

[15] R. Jakob, Instabile Extremalen des Shiffman-Funktionals, Bonner Math. Schriften 362 (2003).

[16] R. Jakob, Unstable extremal surfaces of the "Shiffman-functional", Calc. Var., in press.

[17] R. Jakob, H-Flächen-Index-Formel, Bonner Math. Schriften 366 (2004).

[18] R. Stöcker, H. Zieschang, Algebraische Topologie 2. Auflage, Teubner, 1994.

[19] M. Struwe, On a critical point theory for minimal surfaces spanning a wire in $\mathbb{R}^{3}$, J. Reine Angew. Math. 349 (1984) 1-23.

[20] M. Struwe, Plateau's Problem and the Calculus of Variations, Princeton University Press, 1988.

[21] A.J. Tromba, Degree theory on oriented infinite dimensional varieties and the Morse number of minimal surfaces spanning a curve in $\mathbb{R}^{n}$, Part I: $(n \geqslant 4)$, Trans. Amer. Math. Soc. 290 (1) (1985) 385-413.

[22] A.J. Tromba, Degree theory on oriented infinite dimensional varieties and the Morse number of minimal surfaces spanning a curve in $\mathbb{R}^{3}$, Part II: $(n=3)$, Manuscripta Math. 48 (1984) 139-161. 